\title{
Origin of methane and light hydrocarbons in natural fluid emissions: A key study from Greece
}

\author{
Kyriaki Daskalopoulou ${ }^{\mathrm{a}, \mathrm{b}}$, Sergio Calabrese ${ }^{\mathrm{a}}$, Fausto Grassa ${ }^{\mathrm{c}}$, Konstantinos Kyriakopoulos ${ }^{\mathrm{b}}$, \\ Francesco Parello ${ }^{\mathrm{a}}$, Franco Tassi ${ }^{\mathrm{d}}$, Walter D'Alessandro ${ }^{\mathrm{c}, *}$ \\ ${ }^{a}$ Università degli Studi di Palermo, Dipartimento della Terra e del Mare, via Archirafi, 36, 90123 Palermo, Italy \\ ${ }^{\mathrm{b}}$ National and Kapodistrian University of Athens, Department of Geology and Geoenvironment, Panepistimioupolis, Ano Ilissia, 15784 Athens, Greece \\ ${ }^{\mathrm{c}}$ Istituto Nazionale di Geofisica e Vulcanologia, via Ugo la Malfa 153, 90146 Palermo, Italy \\ ${ }^{\mathrm{d}}$ Università degli Studi di Firenze, Dipartimento della Terra, via G. La Pira 4, 50121 Florence, Italy
}

\section{A R T I C L E I N F O}

Editor: Dong Hailiang

\begin{abstract}
A B S T R A C T
Greece, a country characterised by intense seismic and volcanic activity, has a complex geodynamic and geological setting that favours the occurrence of many gas manifestations. In this study, we address the origin of $\mathrm{CH}_{4}$ and light hydrocarbons in cold and thermal emissions discharging along the Hellenic territory. Also, we investigate their possible relationship with the main geochemical composition of the gases and the different geological settings of the sampling sites. For this purpose we collected 101 new samples that were analysed for their chemical $\left(\mathrm{O}_{2}, \mathrm{~N}_{2}, \mathrm{CH}_{4}, \mathrm{CO}_{2}, \mathrm{He}, \mathrm{Ne}, \mathrm{Ar}, \mathrm{H}_{2}, \mathrm{H}_{2} \mathrm{~S}\right.$ and $\mathrm{C}_{2}-\mathrm{C}_{6}$ hydrocarbons) and isotopic $\left(\mathrm{R} / \mathrm{R}_{\mathrm{A}}, \delta^{13} \mathrm{C}-\mathrm{CO}_{2}\right.$, $\delta^{13} \mathrm{C}-\mathrm{CH}_{4}$ and $\delta^{2} \mathrm{H}-\mathrm{CH}_{4}$ ) composition. Results show that $\mathrm{CH}_{4}$ presents a wide range of concentrations (from $<0.5$ to $925,200 \mu \mathrm{mol} / \mathrm{mol})$ and isotopic values $\left(\delta^{13} \mathrm{C}-\mathrm{CH}_{4}\right.$ from -79.8 to $+45.0 \%$ vs. V-PDB; $\delta^{2} \mathrm{H}-\mathrm{CH}_{4}$ from -311 to $+301 \%$ vs. V-SMOW). Greece was subdivided in four geologic units (External [EH] and Internal [IH] Hellenides, Hellenic Hinterland [HH] and active Volcanic Arc [VA]) and a decreasing $\mathrm{CH}_{4}$ concentration from $\mathrm{EH}$ to $\mathrm{HH}$ was recognized, whereas $\mathrm{CH}_{4}$ showed intermediate concentrations in VA. The $\mathrm{CH}_{4} /\left(\mathrm{C}_{2} \mathrm{H}_{6}+\mathrm{C}_{3} \mathrm{H}_{8}\right)$ ratios (from 1.5 to 93,200), coupled with $\mathrm{CH}_{4}$ isotopic features, suggest that the light alkanes derive from different primary sources and are affected by secondary processes. An almost exclusive biotic, mainly microbial, origin of $\mathrm{CH}_{4}$ can be attributed to $\mathrm{EH}$ gases. Cold gases at $\mathrm{IH}$ have mainly a thermogenic origin, although some gases connected to continental serpentinization may have an abiogenic origin. Methane in gases bubbling in thermal waters of $\mathrm{IH}, \mathrm{HH}$ and VA and fumarolic gases of the VA seem to have an abiogenic origin, although their chemical and isotopic characteristics may have been produced by secondary oxidation of thermogenic $\mathrm{CH}_{4}$, a process that in some of the sampled gases causes extremely positive isotopic values $\left(\delta^{13} \mathrm{C}_{-} \mathrm{CH}_{4}\right.$ up to $+45.0 \%$ vs. V-PDB and $\delta^{2} \mathrm{H}_{-} \mathrm{CH}_{4}$ up to $+301 \%$ vs. V-SMOW).
\end{abstract}

\section{Introduction}

Geogenic emissions of carbon greenhouse gases (mainly $\mathrm{CO}_{2}$ and $\mathrm{CH}_{4}$ ) have a significant impact on the global carbon budget (Kvenvolden, 1993; Klusman and Jakel, 1998; Mörner and Etiope, 2002). Notwithstanding, a reliable estimation of the effective amount discharged from natural manifestations is still a challenge (Guliyev and Feizullayev, 1997; Milkov, 2000 and references therein; Etiope et al., 2009). Methane, the most abundant organic gas compound in Earth's atmosphere, has a potential global warming 28 times higher than that of $\mathrm{CO}_{2}$ on a 100-year time horizon (Ciais et al., 2013; Etiope, 2015). In a natural environment, the production of $\mathrm{CH}_{4}$ involves organic matter either as an active (microbial production) or as a passive agent (thermogenic degradation) (Schoell, 1980, 1988; Whiticar, 1999a, 1999b). Microbial activity by Archaea occurring during the diagenesis of sediments at relatively low temperatures (up to $122^{\circ} \mathrm{C}-$ Takai et al., 2008) predominantly produces methane, subordinately ethane and, likely, trace amounts of propane (Formolo, 2010). On the other hand, thermogenic methane is produced at higher temperatures $\left(>150^{\circ} \mathrm{C}-\right.$ Quingley and MacKenzie, 1988) by the thermal cracking of organic matter (catagenesis) or oil. Thermogenic gases can be either independent from oil reservoirs or associated with them, having variable amounts of ethane, propane, butane, and condensate $\left(\mathrm{C}_{5+}\right.$ higher hydrocarbons).

Abiogenic processes able to synthesize $\mathrm{CH}_{4}$ from inorganic molecules at high temperatures, have been also hypothesized to occur in

\footnotetext{
* Corresponding author.

E-mail address: walter.dalessandro@ingv.it (W. D'Alessandro).
} 
natural environments (Etiope and Sherwood Lollar, 2013). Among others, reduction of graphite (Holloway, 1984) or, thermal decomposition of siderite (McCollom, 2013) has been proposed. However, the main abiotic process is considered to be the reduction of gaseous $\mathrm{CO}$ or $\mathrm{CO}_{2}$ (Berndt et al., 1996; Horita and Berndt, 1999; Foustoukos and Seyfried, 2004). The reduction process occurs mainly through the socalled Fischer-Tropsch-type reactions, which are the Fischer-Tropsch reaction (sensu stricto).

$(2 \mathrm{n}+1) \mathrm{H}_{2}+\mathrm{nCO} \rightarrow \mathrm{C}_{\mathrm{n}} \mathrm{H}_{2 \mathrm{n}+2}+\mathrm{nH}_{2} \mathrm{O}$

that allows also the production of minor amounts of light hydrocarbons and the Sabatier reaction:

$4 \mathrm{H}_{2}+\mathrm{CO}_{2} \rightarrow \mathrm{CH}_{4}+2 \mathrm{H}_{2} \mathrm{O}$

Both reactions have high activation energies but in the presence of catalysts, such as native transition metals like $\mathrm{Fe}, \mathrm{Co}, \mathrm{Cr}$ or $\mathrm{Ni}$ in natural systems, these reactions occur also in the $100-300{ }^{\circ} \mathrm{C}$ range $(\mathrm{McCollom}$, 2013), while recently Etiope and Ionescu (2015) suggested that $\mathrm{CH}_{4}$ can be effectively produced in the temperature range $20-90{ }^{\circ} \mathrm{C}$ in the presence of Ru catalyst within chromite-rich serpentinized rocks.

The Hellenic territory has an intense geodynamic activity, giving rise to (i) the highest seismicity of whole Europe (Burton et al., 2004), (ii) the presence of an active volcanic arc (Pe-Piper and Piper, 2002) and many areas of anomalous high geothermal gradient (Fytikas and Kolios, 1979), and (iii) the widespread occurrence of cold and thermal springs (D'Alessandro and Kyriakopoulos, 2013). As commonly observed in many hydrothermal systems worldwide distributed (e.g., Capaccioni et al., 1993, 2004; Tassi, 2004; Tassi et al., 2005a, 2005b, 2012), the natural fluid discharges in the Hellenic territory contain significant concentrations of $\mathrm{CH}_{4}$ and light hydrocarbons. Although parts of the Hellenic territory have been target for oil and gas exploration, only limited chemical and isotopic data have been published about these gases (Kamberis et al., 2000; Rigakis et al., 2001; Etiope, 2009; Etiope et al., 2013a). Some data exist also for some hydrothermal systems (Fiebig et al., 2009, 2013, 2015; D'Alessandro et al., 2014) and for gases deriving from on-land serpentinization processes (Etiope et al., 2013; D'Alessandro et al., 2017) for the same area. The present work, through a widespread sampling of gas manifestations, constrains the origin of $\mathrm{CH}_{4}$ and the postgenetic modifications that could affect the sampled gases all over Greece. To this end, we collected 101 samples from fumarolic, thermal and cold discharges and analysed the concentrations of major $\left(\mathrm{O}_{2}, \mathrm{~N}_{2}, \mathrm{CH}_{4}, \mathrm{CO}_{2}\right)$ and minor gas species $(\mathrm{He}, \mathrm{Ne}$, Ar, $\mathrm{H}_{2}, \mathrm{H}_{2} \mathrm{~S}, \mathrm{C}_{2} \mathrm{H}_{6}, \mathrm{C}_{3} \mathrm{H}_{8}, \mathrm{C}_{3} \mathrm{H}_{6}, i-\mathrm{C}_{4} \mathrm{H}_{10}, n-\mathrm{C}_{4} \mathrm{H}_{10} \mathrm{C}_{3} \mathrm{H}_{6}, i-\mathrm{C}_{4} \mathrm{H}_{8}$ and $\mathrm{C}_{6} \mathrm{H}_{6}$ ) as well as the carbon isotopic composition of $\mathrm{CH}_{4}$ and $\mathrm{CO}_{2}$, the hydrogen isotopic composition of $\mathrm{CH}_{4}$ and the isotopic composition of He. These data, integrated by literature data (Rigakis et al., 2001; Etiope, 2009; Etiope et al., 2006; Etiope et al., 2013a; 2013b; D'Alessandro et al., 2014, 2017), were used to relate origin and postgenetic processes affecting $\mathrm{CH}_{4}$ to the geolithologic situation of the sampled manifestations.

\subsection{Study area}

The geotectonic evolution of the broader area of Greece has been described as a discontinuous southwestward migration of the Alpine orogenic process with successive subductions of the Tethyan oceanic basins, producing collisional tectonics in the areas between Eurasia and Gondwana during Mesozoic and Tertiary times (Robertson and Dixon, 1984; Mountrakis, 1985, 1986). According to the plate tectonics theory, new palaeogeographic and tectonic models were proposed about the evolution of Tethys and the emplacement of the ophiolites (Dercourt, 1972; Roberts and Koukouvelas, 1996; Bortolotti and Principi, 2005; van Hinsbergen et al., 2005). Based on these models, the Aegean Region was divided into several isopic/structural zones. According to various authors (Smith and Moores, 1974; Mountrakis, 1985, 2010), who described the gradual rifting of various continental fragments of
Gondwana at the beginning of the Mesozoic, their independent motion toward Eurasia that created new oceanic crust to the rear, and their final collision with the Eurasia at the end of the Mesozoic, the structural zones (Fig. 1) from west to east can be subdivided, as follows:

a) External Hellenides: i) Parnassos; ii) Gavrovo-Tripolis; iii) Paxos zones that correspond to a neritic continental sea depositional environment; iv) Ionian zone that stands for a neritic intracontinental basin with pelagic sediments; v) Pindos zone that is formed from sedimentary remnants.

b) Internal Hellenides: i) Circum-Rhodope zone that includes volcanoclastic deposits, sea deposits ending up in deep-sea sediments westwards, flysch and molasses; ii) Vardar/Axios zone that is characterised by deep-sea sediments and the obducted ophiolites; iii) Pelagonian zone consisting of neritic sediments; iv) Subpelagonian zone with the obducted ophiolites; v) Attico-Cycladic zone that is envisaged as a continental fragment having undergone neritic sedimentation.

c) Hellenic Hinterland: i) Rhodope and ii) Serbomacedonian Massifs. Both Massifs represent an old continental crust affected by Alpidic metamorphism and consist of Precambrian-Silurian crystalline rocks (Anders et al., 2006; Reischmann and Kostopoulos, 2007) bearing few neritic deposits and also document Late Eocene - Early Oligocene granitoid intrusions (Fig. 1).

To sum up, the Hellenides are the result of the collision of several microcontinental fragments with the margin of Eurasia through the Cretaceous and Paleogene (van Hinsbergen et al., 2005). After the collision, the Hellenides have been characterised by widespread extension, particularly since the Miocene, as a result of subduction rollback from the oceanic crust of the African plate. The modern fault pattern is a result of the westward and south-westward motion of the Aegean-Anatolian microplate with respect to the Eurasian plate.

The Paleogene Hellenide orogeny of Greece and its eastward continuation into western Turkey resulted from the collision of the Apulian microcontinental fragment in the Eocene to Oligocene with the Pelagonian, Rhodope, and Serbo-Macedonian fragments, which had previously accreted to the southern margin of Eurasia in the Cretaceous. Subsequent extension in the Aegean was rapid, likely due to subduction rollback over residual oceanic crust of the African plate, whereas Anatolia had been bounded by African continental crust south of Cyprus since the Early Miocene. This regional extension and the thermal effects of asthenospheric upwelling, related to changes in the geometry of subducting slabs, have been interpreted as causing magma genesis principally within the lithospheric mantle (Pe-Piper and Piper, 2002). Ophiolites were mostly exposed in the Pindos and Vardar oceanic basins, forming two subparallel ophiolitic zones. Their emplacement took place at the closure of the Mesozoic Neotethys, initially in the Upper Jurassic-Lower Cretaceous and finally during the post-Paleocene times (e.g. Ferrière et al., 2016; Papanikolaou, 2009; Robertson, 2004, 2012; Stampfli et al., 2003). At the south Aegean Volcanic Arc, the activity started during the Upper Pliocene (Fytikas et al., 1986) and is currently active. The calc-alkaline volcanic activity of the Southern Aegean region developed in various volcanic centres from Sousaki to Nisyros through Methana, Milos and Santorini.

\section{Materials and methods}

Names, sampling date and coordinates of all new sampling sites can be found as supplementary material in Table S1. Bubbling gases were sampled using an inverted funnel positioned above the bubbles, whereas soil gases were collected by inserting a pipe in the soil at $>$ $50 \mathrm{~cm}$ depth and driving the gas by a syringe and a 3-way valve. Dry gases were collected in $12 \mathrm{~mL}$ Exetainer ${ }^{\circledR}$ vials (only for hydrocarbon analyses) and in glass flasks equipped with two stopcocks (for the remaining analyses). Dissolved gas samples were collected by using of 

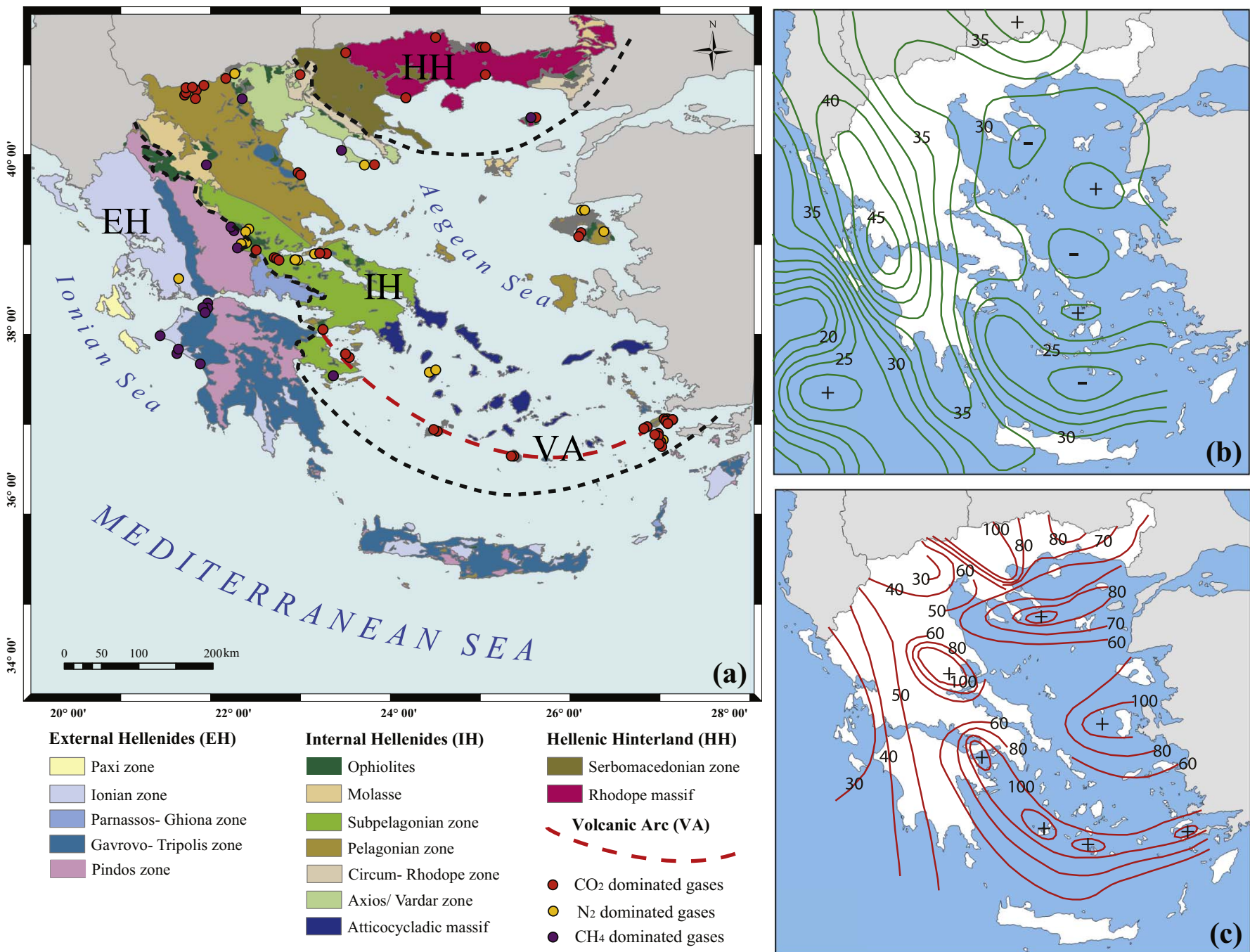

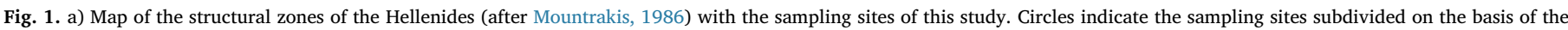

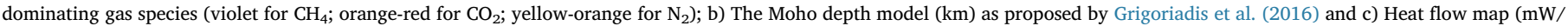
$\mathrm{cm}^{2}$ ) as proposed by Fytikas and Kolios (1979). (For interpretation of the references to colour in this figure legend, the reader is referred to the web version of this article.)

glass vials sealed by gas tight rubber/teflon plugs and analysed applying the "headspace technique" following the method proposed by (Capasso and Inguaggiato, 1998). To allow rapid comparison, dissolved gases have been recalculated as free gas phase in equilibrium with the sampled water. These, expressed in $\mu \mathrm{mol} / \mathrm{mol}$, have been obtained from the partial pressure values taking into account the solubility coefficients of each gas species at laboratory temperature.

In the laboratory, samples were analysed for $\mathrm{He}, \mathrm{H}_{2}, \mathrm{H}_{2} \mathrm{~S}, \mathrm{O}_{2}, \mathrm{~N}_{2}$, $\mathrm{CH}_{4}$ and $\mathrm{CO}_{2}$ by gas-chromatography (Perkin Elmer Clarus500 equipped with a double Carboxen 1000 columns, TCD-FID detectors) using $\mathrm{Ar}$ as the gas carrier. Ar was analysed with a Perkin Elmer XL gaschromatograph with MSieve 5A column, TCD detector having $\mathrm{He}$ as carrier. Analytical uncertainties were $\pm 5 \%$. Hydrocarbon analyses were performed with a Shimadzu 14a gas-chromatograph equipped with a Flame Ionization Detector (FID) using $\mathrm{He}$ as the carrier gas (Vaselli et al., 2006). The analytical error was $\leq 5 \%$.

Carbon isotope composition of $\mathrm{CO}_{2}$ was determined by using a Thermo Delta Plus XP, coupled with a Thermo TRACE Gas Chromatograph (GC) and a Thermo GC/C III interface. The TRACE GC is equipped with a Poraplot $\mathrm{Q}(25 \mathrm{~m} \times 0.32 \mathrm{~mm})$ column and uses Helium (5.6) as carrier gas at a constant flow of $0.9 \mathrm{~cm}^{3} \mathrm{~min}^{-1}$. Undesired gas species, such as $\mathrm{N}_{2}, \mathrm{O}_{2}$, and $\mathrm{CH}_{4}$, are vented to atmosphere by means of back-flush of He and a Sige valve.

The ${ }^{13} \mathrm{C} /{ }^{12} \mathrm{C}$ ratios are reported as $\delta^{13} \mathrm{C}_{\mathrm{CO} 2}$ values with respect to the $\mathrm{V}$ -
PDB standard. Carbon isotope ratios were determined by comparing three in-house standards $\left(\delta^{13} \mathrm{C}\right.$ ranging from $+0.3 \pm 0.1 \%$ to $-28.5 \pm 0.3 \%$ o vs V-PDB calibrated by using of a $\mathrm{CO}_{2}$ standard (RM8564) with known isotopic composition $\left(\delta^{13} \mathrm{C}=-10.45 \pm 0.04 \%\right.$ vs V-PDB) and two international standards (NBS 18 and NBS 19). External precision, computed as $1 \sigma$ (standard deviation) on ten measurements of the same sample, is $0.1 \%$.

Carbon and Hydrogen isotopes of $\mathrm{CH}_{4}$ both in free gases and in dissolved gases were measured using a Thermo TRACE GC interfaced to a Delta Plus XP gas source mass spectrometer and equipped with a Thermo GC/C III (for Carbon) and with GC/TC peripherals (for Hydrogen).

The gas chromatograph was equipped with an Rt-Q Plot column (Restek $30 \mathrm{~m} \times 0.32 \mathrm{~mm}$ i.d.) and the oven was held at a constant temperature $\left(50{ }^{\circ} \mathrm{C}\right.$ for carbon and $40^{\circ} \mathrm{C}$ for Hydrogen). The flow rate of carrier gas (He of 5.6 grade) was held at a constant flux of $0.8 \mathrm{~cm}^{3} \mathrm{~min}^{-1}$. A split/splitless injector with a split ratio from 10:1 to 80:1 was used for sample introduction, except for diluted samples $\left(\mathrm{CH}_{4}\right.$ concentration lower than $10 \mathrm{mmol} / \mathrm{mol}$ ) when direct on-column injection was performed.

The inlet system, better described in Grassa et al. (2010), consists of a stainless steel loop with a known volume $(50 \mu \mathrm{l})$, connected to a twoposition six-port Valco ${ }^{\circledast}$ valve. Before the introduction of the sample, a vacuum of $10^{-2}$ mbar measured with an EBRO pressure gauge is 
ensured by a rotary vane pump. Once $\mathrm{CH}_{4}$ was separated from the gas mixture, it was quantitatively converted to $\mathrm{CO}_{2}$ by passing through a combustion oven $\left(T=940{ }^{\circ} \mathrm{C}\right)$ for ${ }^{13} \mathrm{C} /{ }^{12} \mathrm{C}$ ratios analysis or to $\mathrm{H}_{2}$ by passing it through a reactor set at a temperature of $1440{ }^{\circ} \mathrm{C}$ for ${ }^{2} \mathrm{H} /{ }^{1} \mathrm{H}$ ratios analysis. Each sample analysis took about $500 \mathrm{~s}$.

The ${ }^{13} \mathrm{C} /{ }^{12} \mathrm{C}$ ratios are reported as $\delta^{13} \mathrm{C}-\mathrm{CH}_{4}$ values with respect to the V-PDB standard and ${ }^{2} \mathrm{H} /{ }^{1} \mathrm{H}$ ratios are reported here as $\delta^{2} \mathrm{H}-\mathrm{CH}_{4}$ values with respect to the V-SMOW standard. Carbon isotope ratios were determined by comparing an in-house standard $\left(\delta^{13} \mathrm{C}=-49.5 \pm 0.2 \%\right.$ ) calibrated by using of four $\mathrm{CH}_{4}$ standards (Isometric Instruments) with known isotopic composition $\left(\delta^{13} \mathrm{C}\right.$ ranging from $-23.9 \pm 0.3 \%$ to $-66.5 \pm 0.3 \%$ vs V-PDB).

Hydrogen isotope ratios were determined comparing an in-house standard $\left(\delta^{13} \mathrm{C}=-200 \pm 2.0 \%\right.$ o $)$ with a $\mathrm{CH}_{4}$ standard with known isotopic composition $\left(\delta^{2} \mathrm{H}=-186.1 \pm 3.0 \%\right.$ vs V-SMOW).

External reproducibility, estimated as $1 \sigma$ (standard deviation) on ten measurements of the same sample, is $0.2 \%$ and $2.0 \% 0$ for carbon and hydrogen isotopes respectively.

In $\mathrm{CO}_{2}$-dominated gases having $\mathrm{CH}_{4}$ concentrations lower than $1000 \mu \mathrm{mol} / \mathrm{mol}$, the analyses of the isotope ratios of methane were carried out in the headspace gas samples collected using pre-evacuated $60 \mathrm{~mL}$ glass flasks filled with $20 \mathrm{~mL}$ of a $4 \mathrm{~N} \mathrm{NaOH}$ solution (Giggenbach and Gougel, 1989).

The abundance and isotope composition of $\mathrm{He}$, and the ${ }^{4} \mathrm{He} /{ }^{20} \mathrm{Ne}$ ratios, were determined by separately admitting $\mathrm{He}$ and $\mathrm{Ne}$ into a split flight tube mass spectrometer (Helix SFT). Helium isotope compositions are given as $R / R_{A}$, where $R$ is the $\left({ }^{3} \mathrm{He} /{ }^{4} \mathrm{He}\right)$ ratio of the sample and $\mathrm{R}_{\mathrm{A}}$ is the atmospheric $\left({ }^{3} \mathrm{He} /{ }^{4} \mathrm{He}\right)$ ratio $\left(\mathrm{R}_{\mathrm{A}}=1.386 \times 10^{-6}\right)$. The analytical technique is described more in detail in Paonita et al. (2012). The analytical errors were generally $<1 \%$.

\section{Results}

\subsection{Chemical composition of the gases}

On the basis of the spatial distribution of the gas discharges and their type of emission, the whole dataset was subdivided into 4 main geological domains, as follows: 1) External Hellenides (EH) - 17 samples; 2) Internal Hellenides (IH) - 60 samples; 3) Hellenic Hinterland (HH) - 8 samples; 4) Volcanic Arc (VA) - 33 samples. The chemical composition of the dry gas fraction $\left(\mathrm{CO}_{2}, \mathrm{H}_{2} \mathrm{~S}, \mathrm{CH}_{4}, \mathrm{~N}_{2}, \mathrm{O}_{2}, \mathrm{Ar}\right.$, $\mathrm{He}$ and $\mathrm{H}_{2}$ ) of the 101 gas samples analysed in the present study is reported in Table 1, whereas the 17 literature data were taken from Rigakis et al., 2001, Etiope, 2009, Etiope et al., 2006; Etiope et al., 2013a, 2013b, D'Alessandro et al., 2014, 2017. Dry gases were dominated by either $\mathrm{CO}_{2}$ (from 18 to $997,000 \mu \mathrm{mol} / \mathrm{mol}$ ) or $\mathrm{N}_{2}$ (from 1100 to $989,000 \mu \mathrm{mol} / \mathrm{mol}$ ) or $\mathrm{CH}_{4}$ (from $<0.5$ to $913,000 \mu \mathrm{mol} / \mathrm{mol}$ ) gas species (Fig. 2a). $\mathrm{O}_{2}$ and Ar showed concentrations up to 177,000 and $12,000 \mu \mathrm{mol} / \mathrm{mol}$ respectively. $\mathrm{H}_{2} \mathrm{~S}$ was either below detection limit or showing concentrations generally lower than $10,000 \mu \mathrm{mol} / \mathrm{mol}$ except for the fumaroles of Nisyros, where the concentrations reached up to $192,000 \mu \mathrm{mol} / \mathrm{mol}$ (Fiebig et al., 2013). He concentrations were up to $2240 \mu \mathrm{mol} / \mathrm{mol}$.

In the $\mathrm{CO}_{2}-\mathrm{CH}_{4}-\mathrm{N}_{2}$ ternary diagram samples collected in the $\mathrm{EH}$ show $\mathrm{CH}_{4}$ (thermal emissions) and $\mathrm{CH}_{4}-\mathrm{N}_{2}$ dominated gases (cold emissions) with relative low contents of $\mathrm{CO}_{2}(25-34,000 \mu \mathrm{mol} / \mathrm{mol})$. Gases collected from the thermal manifestations of the $\mathrm{IH}$ are rich in $\mathrm{N}_{2^{-}}$ $\mathrm{CO}_{2}$, whereas in the remaining cold manifestations the $\mathrm{CO}_{2}$-rich gases are prevailing although sometimes they showed relatively high concentrations of $\mathrm{N}_{2}$ (samples 8, 17, 18, 28, 29, 89 and 90). In this area, three $\mathrm{CH}_{4}$-dominated gases (samples 1,50 and 82, with 880,000, 764,000 and $576,000 \mu \mathrm{mol} / \mathrm{mol}$ respectively) were also found. In $\mathrm{HH}$, $\mathrm{CO}_{2}$ is the most abundant gas species, except for samples 78 and 79 that have dominant $\mathrm{N}_{2}(537,000$ and $417,000 \mu \mathrm{mol} / \mathrm{mol}$ respectively). The thermal emissions of the VA are $\mathrm{CO}_{2}$-dominated, except sample 86 that is showing relatively high $\mathrm{N}_{2}(368,000 \mu \mathrm{mol} / \mathrm{mol})$. Most gases collected from the cold emissions of the same region show the same chemical features as the gases discharged from the thermal springs, with the exception of samples 67, 73, 74, 87 and 102 that were $\mathrm{N}_{2}$-dominated.

The He- $\mathrm{N}_{2}$-Ar ternary diagram (Giggenbach, 1996) (Fig. 2b) suggests important mixing processes between deep crustal or mantle gases and also gases originating in the upper crust or atmosphere. The $\mathrm{N}_{2} / \mathrm{Ar}$ ratio can be used to distinguish the relative contributions to a gas mixture from air, air-saturated waters, and fluids deriving from interactions within subducting and crustal rocks/sediments and mantle gases (e.g. Snyder et al., 2001, 2003). $\mathrm{CH}_{4}-\mathrm{N}_{2}$ rich gases of the $\mathrm{EH}$ and some of the $\mathrm{CO}_{2}$ dominated cold emissions of the $\mathrm{IH}$ plot close to the Air and Air Saturated Water (ASW) points, indicating a dominant atmospheric component for these gases. Some of the samples from VA and the cold emissions of $\mathrm{IH}\left(\mathrm{CO}_{2}\right.$ dominated) fall on the theoretical line that connects ASW with a He-rich source with a relatively low contribution of crustal $\mathrm{N}_{2}$ (Giggenbach, 1996). Samples of the HH rich in $\mathrm{CO}_{2}$ and some of the emissions that occur in the $\mathrm{IH}$, characterised by a $\mathrm{N}_{2}-\mathrm{CO}_{2}$ composition, show atmospheric $\mathrm{N}_{2}$ mixed with non-atmospheric He.

\subsection{Isotope composition of the gases}

The $\delta^{13} \mathrm{C}-\mathrm{CO}_{2}$ values ranged from -20.1 to $+8.5 \%$ although most of them are comprised in a narrower isotopic range from -7.5 to $+0.5 \%$ (Table 2). Samples from $\mathrm{EH}$ have negative values, from -14.1 to $-7 \%$. The $\delta^{13} \mathrm{C}-\mathrm{CO}_{2}$ values of the gases collected from $\mathrm{IH}$, are in a wide range (from $-16 \%$ to $+8.5 \%$ ). In $\mathrm{HH}$, they show a narrower range $(-2.4$ to $+0.3 \%$ ), whereas in VA they range from -3.5 to $0.4 \%$, apart from the sample 67 whose $\delta^{13} \mathrm{C}^{-} \mathrm{CO}_{2}$ value was $-20.1 \%$. Gases collected from emissions of $\mathrm{IH}$ and $\mathrm{HH}$, in which $\mathrm{N}_{2}-\mathrm{CO}_{2}$ are the dominant species, show a range of values of $\delta^{13} \mathrm{C}-\mathrm{CO}_{2}$ that vary between -16 to $-0.9 \%$ and -1.9 to $0.3 \%$, respectively.

$\mathrm{CO}_{2}$ dominated gases from VA show relatively high values for the isotopic ratio of $\mathrm{He}$ (from 0.21 to $6.71 \mathrm{R} / \mathrm{R}_{\mathrm{A}}$; Table 2), whereas cold and thermal gases that occur in $\mathrm{HH}$ and $\mathrm{IH}$ are characterised by values that reach up to 0.41 and $1.27 \mathrm{R} / \mathrm{R}_{\mathrm{A}}$, respectively (Table 2). The $\mathrm{N}_{2}-\mathrm{CH}_{4}$ dominated gas samples from EH show values from 0.08 to $0.41 \mathrm{R} / \mathrm{R}_{\mathrm{A}}$, whereas the $\mathrm{N}_{2}$-rich cold manifestations from $\mathrm{IH}$ show values in the range of $0.3-0.97 \mathrm{R} / \mathrm{R}_{\mathrm{A}}$.

\subsection{Hydrocarbons}

\subsubsection{Chemical composition of the hydrocarbons}

Gases from EH show the highest concentrations of $\mathrm{CH}_{4}$, having a median of $721,200 \mu \mathrm{mol} / \mathrm{mol}$ (from 85,000 to $925,000 \mu \mathrm{mol} / \mathrm{mol}$ ). In $\mathrm{IH}$, they present a median of $2600 \mu \mathrm{mol} / \mathrm{mol}$ with values ranging from $<0.5$ up to $880,000 \mu \mathrm{mol} / \mathrm{mol}$, whereas the lowest concentrations are found in $\mathrm{HH}$ with a median of $545 \mu \mathrm{mol} / \mathrm{mol}$ (from 23 up to $879 \mu \mathrm{mol} / \mathrm{mol}$ ). Finally, those collected in VA present a median of $1460 \mu \mathrm{mol} / \mathrm{mol}$ (from 5 up to $117,000 \mu \mathrm{mol} / \mathrm{mol}$ ).

\subsubsection{Isotopic composition of $\mathrm{CH}_{4}$}

The $\delta^{13} \mathrm{C}$ and $\delta^{2} \mathrm{H}$ values of $\mathrm{CH}_{4}$ range from -79.8 to $+45.0 \%$ for $\delta^{13} \mathrm{C}^{-} \mathrm{CH}_{4}$ and from -311 to $+301 \%$ for $\delta^{2} \mathrm{H}-\mathrm{CH}_{4}$, respectively (Table 2). Gases from EH have $\delta^{13} \mathrm{C}_{-} \mathrm{CH}_{4}$ and $\delta^{2} \mathrm{H}-\mathrm{CH}_{4}$ values ranging from -79.8 to $-31.3 \%$ and from -248 to $-62 \%$, respectively. In IH, the values of $\delta^{13} \mathrm{C}$ range from -64 up to $+45 \%$, whereas $\delta^{2} \mathrm{H}$ shows a wide range of values (from-311 to $+301 \%$ ). The $\delta^{13} \mathrm{C}_{-}-\mathrm{CH}_{4}$ vales of the $\mathrm{HH}$ gases range from -34 up to $+1 \%$, whereas their $\delta^{2} \mathrm{H}$ values, apart from sample $75(-174 \%)$, were not measured due to the too low concentrations. The gases from VA show $\delta^{13} \mathrm{C}_{-} \mathrm{CH}_{4}$ values ranging from -38 up to $+2 \%$ and $\delta^{2} \mathrm{H}$ values from -135 to $+36 \%$. 
Table 1

Chemical composition of the gases.

\begin{tabular}{|c|c|c|c|c|c|c|c|c|c|}
\hline N. & Sample & $\mathrm{He}$ & $\mathrm{H}_{2}$ & $\mathrm{O}_{2}$ & $\mathrm{~N}_{2}$ & $\mathrm{CH}_{4}$ & $\mathrm{CO}_{2}$ & $\mathrm{H}_{2} \mathrm{~S}$ & $\mathrm{Ar}$ \\
\hline & & $\mu \mathrm{mol} / \mathrm{mol}$ & $\mu \mathrm{mol} / \mathrm{mol}$ & $\mu \mathrm{mol} / \mathrm{mol}$ & $\mu \mathrm{mol} / \mathrm{mol}$ & $\mu \mathrm{mol} / \mathrm{mol}$ & $\mu \mathrm{mol} / \mathrm{mol}$ & $\mu \mathrm{mol} / \mathrm{mol}$ & $\mu \mathrm{mol} / \mathrm{mol}$ \\
\hline 1 & Katakali & 4.0 & 11 & 589 & 82,500 & 880,000 & 25,000 & $<5$ & 1640 \\
\hline 2 & Psoroneria & 887 & $<0.5$ & 100 & 427,000 & 1120 & 573,000 & $<10$ & 3940 \\
\hline 3 & Psoroneria & 487 & $<2$ & 1700 & 336,000 & 631 & 658,000 & $<5$ & 3900 \\
\hline 4 & Thermopyles & 322 & $<2$ & 59 & 218,000 & 1010 & 760,000 & $<5$ & 1980 \\
\hline 5 & Thermopyles & 240 & $<2$ & 709 & 176,000 & 859 & 831,000 & $<5$ & 1910 \\
\hline 6 & Koniavitis & 581 & $<5$ & 50 & 701,000 & 2090 & 281,000 & 2200 & 8340 \\
\hline 7 & Kammena Vourla & 700 & 3.5 & 100 & 644,000 & 3360 & 352,000 & $<10$ & 6050 \\
\hline 8 & Kammena Vourla & 727 & $<5$ & 218 & 588,000 & 4070 & 402,000 & $<5$ & 5940 \\
\hline 9 & Kallydica & 325 & $<2$ & 161,000 & 508,000 & 1090 & 332,000 & $<5$ & n.d. \\
\hline 10 & Gialtra & 112 & $<5$ & 137,000 & 765,000 & 302 & 44,000 & n.d. & n.d. \\
\hline 11 & Thermopotamos & 0.4 & $<5$ & 1200 & 2800 & 27 & 977,000 & 48 & 40 \\
\hline 12 & Thermopotamos & 1.5 & $<2$ & 4700 & 18,400 & 1200 & 958,500 & $<5$ & 275 \\
\hline 13 & Patra & 1.7 & 50 & 301 & 192,000 & 704,000 & 11,100 & $<5$ & 3500 \\
\hline 14 & Sousaki well & 1.3 & 10 & 1700 & 7500 & 33 & 975,000 & 2500 & 95 \\
\hline 15 & Sousaki cave & 33.0 & $<5$ & 4100 & 37,400 & 10,100 & 937,000 & 511 & 279 \\
\hline 16 & Ilion & 100 & $<5$ & 940 & 78,900 & 1820 & 899,000 & $<5$ & 733 \\
\hline 17 & Ilion & 100 & 3.7 & 1100 & 79,400 & 1800 & 901,000 & $<5$ & 742 \\
\hline 18 & Ilion sea & 199 & $<2$ & 11,300 & 271,000 & 1040 & 694,000 & $<5$ & 5030 \\
\hline 19 & Pausanias & $<5$ & $<5$ & 2300 & 12,900 & 53 & 983,000 & $<5$ & 222 \\
\hline 20 & Pausanias sea & $<5$ & $<5$ & 6100 & 14,500 & 32 & 984,000 & $<5$ & 214 \\
\hline 21 & Thiafi sea & 22.0 & $<2$ & 34,100 & 103,000 & 916 & 832,000 & $<5$ & n.d. \\
\hline 22 & Agia paraskevi sea & 3.1 & 21.0 & 10,600 & 27,000 & 470 & 964,000 & $<5$ & 458 \\
\hline 23 & Agia paraskevi sea 2 & 92.0 & 100 & 3900 & 40,500 & 11,000 & 946,000 & 3100 & 555 \\
\hline 24 & Xyna & 91.0 & 1.6 & 13,000 & 49,600 & 215 & 943,000 & $<5$ & 815 \\
\hline 25 & PPG-1 & 0.4 & $<2$ & 50 & 40,100 & 913,000 & 21,400 & $<5$ & 1180 \\
\hline 26 & Soulanta & 31.0 & 22 & 125 & 350,000 & 649,000 & 25 & n.d. & n.d. \\
\hline 27 & Smokovo & 100 & 27 & 272 & 124,000 & 850,000 & 25 & n.d. & n.d. \\
\hline 28 & Ekkara & 4.0 & 16 & 177,000 & 750,000 & 5720 & 25 & n.d. & n.d. \\
\hline 29 & Ekkara creek & 21.0 & 11 & 2500 & 888,000 & 77,700 & 552 & $<5$ & 10,200 \\
\hline 30 & Ypati & 14.0 & 0.6 & 104 & 42,400 & 4460 & 934,000 & 1260 & 742 \\
\hline 31 & Loutra Ypatis & 105 & $<2$ & 14,500 & 76,300 & 5810 & 888,000 & 385 & 848 \\
\hline 32 & Leonidas & 275 & $<2$ & 9200 & 242,000 & 799 & 726,000 & $<5$ & 2600 \\
\hline 33 & Kokkinonero & 1.6 & $<5$ & 2500 & 7200 & 249 & 955,000 & n.d. & 195 \\
\hline 34 & Kokkinonero 2 & 209 & $<5$ & 4300 & 382,000 & 867 & 581,000 & n.d. & 5040 \\
\hline 35 & Edipsos stadium & $<4$ & $<2$ & 525 & 3000 & 689 & 967,000 & $<5$ & 57 \\
\hline 36 & Ag. Paraskevi & 4.3 & $<5$ & 158 & 23,500 & 521 & 929,000 & 17,400 & 502 \\
\hline 37 & Giatsovo soil & 12.5 & 2.0 & 760 & 20,800 & 2690 & 987,000 & $<5$ & 622 \\
\hline 38 & Analipsi & 19.2 & $<2$ & 4600 & 29,600 & 2350 & 952,000 & $<5$ & 128 \\
\hline 39 & Tropeouchos & 29.0 & $<5$ & 76 & 20,000 & 6200 & 952,000 & n.d. & 233 \\
\hline 40 & Mesochori gas & 4.0 & 1.5 & 1000 & 6500 & 479 & 985,000 & $<5$ & 42 \\
\hline 41 & Mesochori 3 & 1.1 & $<5$ & 50 & 2100 & 214 & 975,000 & $<5$ & 29 \\
\hline 42 & Synergio & 0.4 & $<5$ & 187 & 1100 & $<0.5$ & 997,000 & $<5$ & 24 \\
\hline 43 & Sarri & 0.3 & $<5$ & 93 & 1400 & 95 & 983,000 & n.d. & 62 \\
\hline 44 & Itea & 36.0 & 1.4 & 5700 & 38,300 & 2600 & 951,000 & $<5$ & 223 \\
\hline 45 & Pozar & 474 & $<5$ & 56,200 & 617,000 & $<1$ & 323,000 & $<5$ & 7660 \\
\hline 46 & Promachoi & 223 & $<5$ & 54,400 & 827,000 & 2.0 & 108,000 & n.d. & 9090 \\
\hline 47 & Loutrochori 2 & 374 & $<5$ & 175 & 510,000 & 436,000 & 32,400 & $<5$ & 6290 \\
\hline 48 & Xino Nero (Kilkis) & $<5$ & $<5$ & 2900 & 15,200 & 23 & 965,000 & $<5$ & 304 \\
\hline 49 & Xino Nero 2 (Kilkis) & 6.0 & $<5$ & 7300 & 26,000 & 82 & 939,000 & $<5$ & n.d. \\
\hline 50 & Sani & 762 & 12 & 330 & 226,000 & 764,000 & 1500 & $<5$ & 870 \\
\hline 51 & Thermopigi & 15.0 & $<5$ & 639 & 13,300 & 669 & 967,000 & $<5$ & 218 \\
\hline 52 & Eleftheres & 31.0 & $<2$ & 690 & 72,600 & 703 & 921,000 & $<5$ & 1180 \\
\hline 53 & Polychnitos & 192 & $<5$ & 3200 & 193,000 & 32,800 & 766,000 & $<5$ & 4690 \\
\hline 54 & Lisvori & 471 & $<5$ & 5200 & 519,000 & 20,500 & 444,000 & $<5$ & 7300 \\
\hline 55 & Eftalou & 1370 & $<5$ & 14,400 & 948,000 & 219 & 27,200 & $<5$ & 10,400 \\
\hline 56 & Skala Sikaminias & 1280 & 134 & 1400 & 657,000 & 70,500 & 259,000 & $<5$ & 10,800 \\
\hline 57 & Kolpos Geras & 1020 & $<5$ & 16,100 & 922,000 & 10 & 41,200 & $<5$ & 9780 \\
\hline 58 & Milos Skinopi & 63.0 & $<5$ & 16,700 & 73,700 & 4630 & 829,000 & n.d. & n.d. \\
\hline 59 & Milos DEH & 8.0 & $<5$ & 2200 & 9600 & 818 & 978,000 & n.d. & n.d. \\
\hline 60 & Kefalos & 18.0 & 27 & 3290 & 25,100 & 23,900 & 946,000 & $<5$ & 105 \\
\hline 61 & Kos Paradise & 14.0 & $<2$ & 3100 & 8600 & 12,100 & 959,000 & $<5$ & 170 \\
\hline 62 & Kos Kokkinonero & 5.0 & 3.1 & 3200 & 13,600 & 2970 & 978,000 & $<5$ & 171 \\
\hline 63 & KKN1 & 3.3 & 295 & 3500 & 24,400 & 2400 & 980,000 & 673 & n.d. \\
\hline 64 & Kos Kokkinonero 2 & 4.3 & 30 & 2700 & 8300 & 3140 & 991,000 & $<5$ & n.d. \\
\hline 65 & KVO2 & 34.0 & 1980 & 10,100 & 75,400 & 21,000 & 877,000 & 54 & n.d. \\
\hline 66 & KVO52 & 44.0 & 2.9 & 317 & 4000 & 23,100 & 947,000 & 851 & 31 \\
\hline 67 & Ag. Irini 2 & 446 & $<2$ & 2210 & 989,000 & 6240 & 900 & $<5$ & 7520 \\
\hline 68 & Ag. Irini 1 & 0.9 & $<2$ & 3900 & 9200 & 24 & 963,000 & 15 & 200 \\
\hline 69 & Gyali nord & 0.5 & 3.7 & 2600 & 9600 & 5.0 & 966,000 & $<5$ & 266 \\
\hline 70 & Gyali & 9.0 & $<2$ & 2200 & 23,300 & 68 & 956,000 & $<5$ & 354 \\
\hline 71 & Gyali lake & 21.0 & $<2$ & 716 & 139,000 & 525 & 869,000 & 3800 & n.d. \\
\hline 72 & Gyali west & 5.0 & 31 & 1570 & 8300 & 21,000 & 970,000 & $<5$ & 71 \\
\hline
\end{tabular}


Table 1 (continued)

\begin{tabular}{|c|c|c|c|c|c|c|c|c|c|}
\hline N. & Sample & $\mathrm{He}$ & $\mathrm{H}_{2}$ & $\mathrm{O}_{2}$ & $\mathrm{~N}_{2}$ & $\mathrm{CH}_{4}$ & $\mathrm{CO}_{2}$ & $\mathrm{H}_{2} \mathrm{~S}$ & $\mathrm{Ar}$ \\
\hline & & $\mu \mathrm{mol} / \mathrm{mol}$ & $\mu \mathrm{mol} / \mathrm{mol}$ & $\mu \mathrm{mol} / \mathrm{mol}$ & $\mu \mathrm{mol} / \mathrm{mol}$ & $\mu \mathrm{mol} / \mathrm{mol}$ & $\mu \mathrm{mol} / \mathrm{mol}$ & $\mu \mathrm{mol} / \mathrm{mol}$ & $\mu \mathrm{mol} / \mathrm{mol}$ \\
\hline 73 & Katsouni & 120 & $<2$ & 11,500 & 855,000 & 134 & 155,400 & $<5$ & 11,500 \\
\hline 74 & Lies & 135 & $<2$ & 18,800 & 643,000 & 140 & 315,200 & $<5$ & 9790 \\
\hline 75 & Nea Kessani & 26.0 & $<2$ & 5900 & 14,700 & 386 & 987,000 & $<5$ & 208 \\
\hline 76 & Eleftheres Ag. Marina & 51.0 & $<2$ & 3400 & 180,000 & 756 & 809,300 & $<5$ & 2760 \\
\hline 77 & Paranesti 2 & $<5$ & $<5$ & 1700 & 8700 & 102 & 963,200 & $<5$ & n.d. \\
\hline 78 & Thermes (Xanthi) & 2240 & $<5$ & 9700 & 537,000 & 844 & 467,000 & $<5$ & 8090 \\
\hline 79 & Thermes 3 (Xanthi) & 1570 & $<5$ & 588 & 417,000 & 879 & 573,900 & $<5$ & 7350 \\
\hline 80 & Thermia (Kos) & 2.4 & $<5$ & 1100 & 8900 & 51 & 994,600 & $<5$ & 211 \\
\hline 81 & Thermia sea (Kos) & 16.0 & $<2$ & 2500 & 42,400 & 278 & 955,000 & $<5$ & 98 \\
\hline 82 & Therma Limani & 0.10 & 15 & 53 & 7900 & 576,000 & 363,000 & $<5$ & 150 \\
\hline 83 & Geotrisi & 128 & 97 & 1200 & 30,200 & 94,900 & 857,000 & $<5$ & 624 \\
\hline 84 & Ag. Anargyroi Kithnos & 1100 & $<2$ & 2800 & 664,000 & 833 & 329,000 & $<5$ & 8420 \\
\hline 85 & Kolona & 726 & $<2$ & 26,800 & 836,000 & 0.90 & 136,000 & $<5$ & 8400 \\
\hline 86 & Nea Kameni & 6.0 & 8100 & 83,600 & 367,000 & 264 & 526,000 & $<5$ & 4740 \\
\hline 87 & Erinia (N. Kameni) & 245 & $<2$ & 42,800 & 651,000 & 1300 & 301,000 & $<5$ & 9510 \\
\hline 88 & Ag. Anargyroi & $<5$ & 48 & 14,700 & 957,000 & 27,000 & 1360 & n.d. & n.d. \\
\hline 89 & Ag. Anargyroi 2 & $<5$ & 17 & 18,200 & 779,000 & 203,000 & 250 & n.d. & n.d. \\
\hline 90 & Archani & 12.9 & $<10$ & 7420 & 948,000 & 44,700 & 18 & n.d. & n.d. \\
\hline 91 & Lysimachia & 9.9 & $<10$ & 978 & 602,000 & 363,000 & 34,800 & n.d. & n.d. \\
\hline 92 & Kaitsa & 123 & $<10$ & 3070 & 655,000 & 316,000 & 26,200 & 300 & n.d. \\
\hline 93 & Amplas & 83.4 & 27 & 1780 & 199,000 & 800,000 & 29 & n.d. & n.d. \\
\hline 94 & Platystomo & 345 & $<10$ & 907 & 860,000 & 128,000 & 10,700 & n.d. & n.d. \\
\hline 95 & PP9S Nisyros & 16.0 & 14,800 & 100 & 2500 & 2520 & 779,000 & 173,000 & n.d. \\
\hline 96 & S4 Nisyros & 20.0 & 8240 & 581 & 6500 & 7510 & 822,000 & 151,000 & n.d. \\
\hline 97 & A13 Nisyros & 21.0 & 9540 & 100 & 1390 & 1460 & 813,000 & 175,000 & n.d. \\
\hline 98 & S15 Nisyros & 19.0 & 8050 & 700 & 21,900 & 4350 & 769,000 & 192,000 & n.d. \\
\hline 99 & K7 Nisyros & 10.0 & 6670 & 695 & 6300 & 32,400 & 891,000 & 47,600 & n.d. \\
\hline 100 & Thermia (Kos) & 2.5 & $<2$ & 535 & 9300 & 64 & 989,000 & $<5$ & 98 \\
\hline 101 & Ag. Irini 2 & 412 & $<2$ & 2000 & 613,000 & 117,000 & 256,000 & $<5$ & n.d. \\
\hline
\end{tabular}

n.d. = not determined.

\section{Discussion}

\subsection{Origin of the inorganic gases}

Nitrogen, one of the main components found in the gas samples, shows generally decreasing concentrations from the $\mathrm{EH}$ and $\mathrm{IH}$ to the HH (Fig. 3a). Gases from the VA display on average even lower contents (Fig. 3a). As previously seen in the He- $\mathrm{N}_{2}$-Ar ternary diagram (Fig. 2b), it mostly originates from the atmosphere through the meteoric recharge or through diffusion in the shallowest soil layers. From the same diagram a contribution from crustal sources or from the subducting slab is apparent. At the moment only few $\mathrm{N}$-isotopic values of $\mathrm{N}_{2}$ have been published (Grassa et al., 2010) confirming significant (about 60\%) sedimentary contribution.

In Fig. $4 \mathrm{a}$ it is noticeable that most of the $\mathrm{CO}_{2}$-rich cold manifestations from IH plot close to the atmospheric point suggesting an important atmospheric contribution for He. Gases from EH present in their majority a crustal origin for He whereas those from VA indicate a strong mantle contribution. Most $\mathrm{N}_{2}-\mathrm{CO}_{2}$ dominated gases from IH seem to be fed by a mixed mantle-crustal source with a mantle component

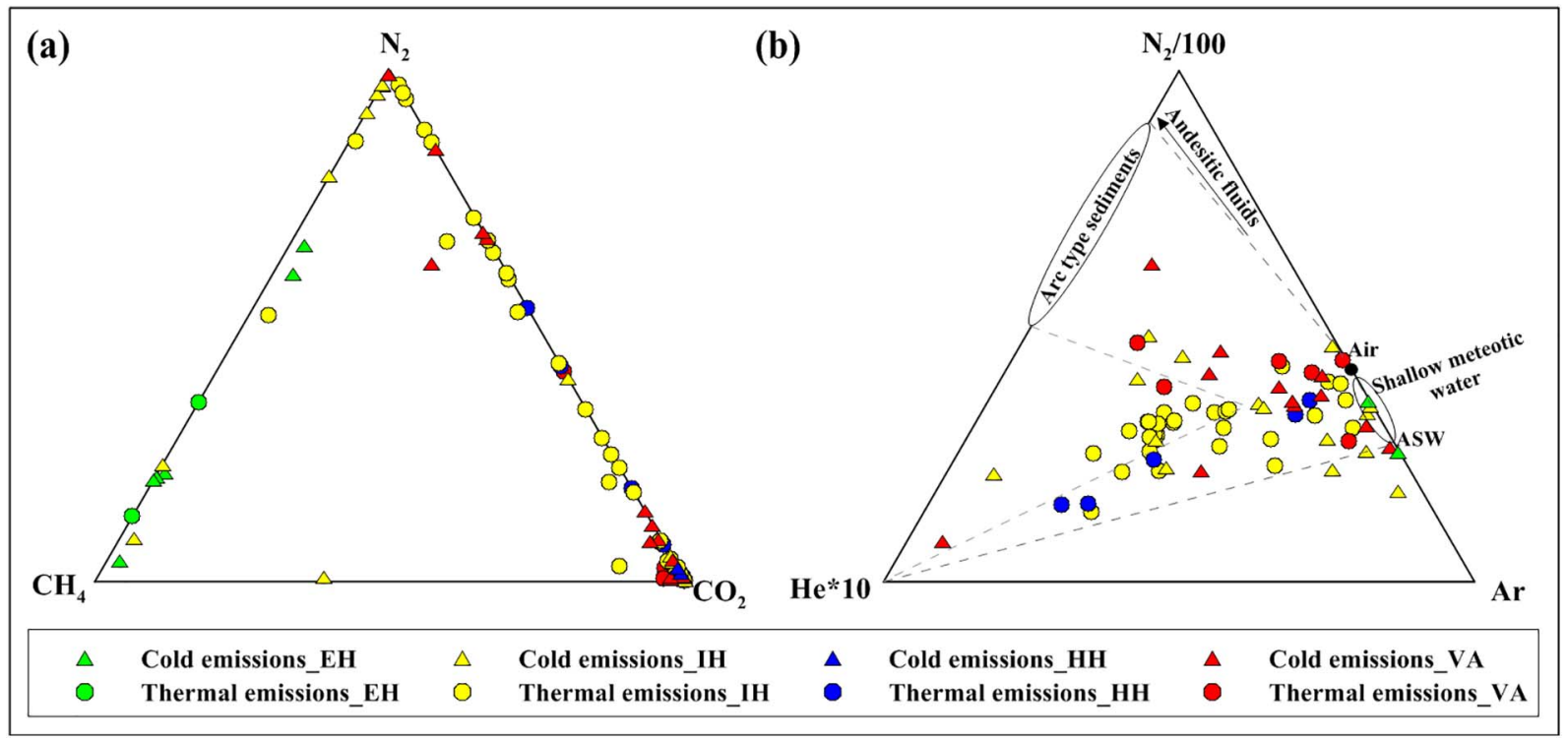

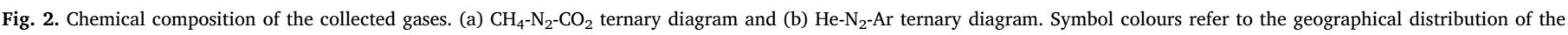
samples, while shape refers to the type of the emission (cold or thermal). 
Table 2

Isotopic composition of the gases.

\begin{tabular}{|c|c|c|c|c|c|}
\hline N. & $\delta^{13} \mathrm{C}\left(\mathrm{CO}_{2}\right)$ & $\delta^{13} \mathrm{C}\left(\mathrm{CH}_{4}\right)$ & $\delta^{2} \mathrm{H}\left(\mathrm{CH}_{4}\right)$ & $\mathrm{R} / \mathrm{R}_{\mathrm{A}}$ & ${ }^{4} \mathrm{He} /{ }^{20} \mathrm{Ne}$ \\
\hline & $\%$ & $\%$ & $\%$ & & \\
\hline 1 & 8.5 & -64.3 & -216 & n.d. & n.d. \\
\hline 2 & n.d. & -2.3 & 129 & 0.07 & 281 \\
\hline 3 & -5.4 & 10.9 & 301 & 0.07 & 95.1 \\
\hline 4 & -5.3 & 1.4 & 30 & 0.20 & 228 \\
\hline 5 & -5.3 & -4.2 & 79 & 0.20 & 208 \\
\hline 6 & -9.8 & -10.6 & n.d. & n.d. & n.d. \\
\hline 7 & -7.4 & 6.3 & 62 & 0.53 & 159 \\
\hline 8 & -7.5 & 4.6 & 60 & 0.51 & 106 \\
\hline 9 & -13.3 & 5.3 & n.d. & n.d. & n.d. \\
\hline 10 & n.d. & n.d. & n.d. & 0.46 & 23.8 \\
\hline 11 & -3.1 & -21.4 & -115 & 0.43 & 8.5 \\
\hline 12 & -2.7 & -21.7 & -124 & 0.44 & 3.2 \\
\hline 13 & -14.1 & -79.8 & -211 & 0.39 & 0.59 \\
\hline 14 & -1.2 & -20.9 & -117 & 0.46 & 8.6 \\
\hline 15 & -1.8 & -21.3 & -114 & 0.21 & 76.7 \\
\hline 16 & -2.6 & -1.4 & 57 & 0.26 & 5.4 \\
\hline 17 & -2.5 & 3.2 & 32 & 0.25 & 252 \\
\hline 18 & -2.6 & 45.0 & n.d. & n.d. & n.d. \\
\hline 19 & -2.9 & n.d. & n.d. & n.d. & n.d. \\
\hline 20 & n.d. & n.d. & n.d. & n.d. & n.d. \\
\hline 21 & -3.4 & -38.6 & n.d. & n.d. & n.d. \\
\hline 22 & -2.0 & -5.6 & -49 & 0.79 & 0.61 \\
\hline 23 & -2.2 & -5.0 & -68 & 0.74 & 308 \\
\hline 24 & -2.5 & n.d. & n.d. & n.d. & n.d. \\
\hline 25 & -7.0 & -74.5 & -210 & 0.41 & 0.77 \\
\hline 26 & n.d. & -60.8 & -248 & 0.07 & 7.8 \\
\hline 27 & n.d. & -44.9 & -196 & 0.04 & 44.0 \\
\hline 28 & n.d. & -33.8 & -276 & 0.34 & 2.0 \\
\hline 29 & n.d. & -33.5 & -234 & 0.30 & 1.0 \\
\hline 30 & -3.2 & -38.7 & n.d. & 0.13 & 6.3 \\
\hline 31 & -4.4 & -32.9 & -105 & 0.05 & 122 \\
\hline 32 & -6.0 & n.d. & n.d. & n.d. & n.d. \\
\hline 33 & -0.8 & -19.8 & -37 & 0.23 & 2.3 \\
\hline 34 & n.d. & n.d. & n.d. & n.d. & n.d. \\
\hline 35 & -3.0 & n.d. & n.d. & n.d. & n.d. \\
\hline 36 & -6.7 & -3.8 & n.d. & 0.74 & 5.9 \\
\hline 37 & -0.6 & -25.7 & -151 & 0.47 & 189 \\
\hline 38 & -0.7 & -23.4 & -145 & 0.23 & 29.8 \\
\hline 39 & -1.2 & -29.4 & -187 & n.d. & n.d. \\
\hline 40 & -1.3 & -21.0 & -131 & 0.62 & 5.1 \\
\hline 41 & -0.4 & -22.5 & n.d. & 0.66 & 50.7 \\
\hline 42 & -0.8 & n.d. & n.d. & 0.57 & 1.4 \\
\hline 43 & n.d. & n.d. & n.d. & 0.56 & 1.6 \\
\hline 44 & 0.5 & -27.7 & -157 & 0.30 & 15.3 \\
\hline 45 & -5.4 & n.d. & n.d. & 0.82 & 44.5 \\
\hline 46 & -7.2 & n.d. & n.d. & 1.27 & 30.3 \\
\hline 47 & -16.0 & -18.8 & -113 & n.d. & n.d. \\
\hline 48 & -2.4 & 1.1 & n.d. & 0.26 & 14.8 \\
\hline 49 & n.d. & n.d. & n.d. & n.d. & n.d. \\
\hline 50 & -9.4 & -57.0 & -180 & 0.68 & 1490 \\
\hline 51 & -2.1 & -1.1 & n.d. & 0.65 & 107 \\
\hline 52 & -1.1 & -23.5 & n.d. & 0.41 & 36.5 \\
\hline 53 & -2.0 & -19.9 & -106 & 0.82 & 82.2 \\
\hline 54 & -3.9 & -7.2 & -21 & 0.85 & 97.0 \\
\hline 55 & -11.8 & n.d. & n.d. & 0.83 & 103 \\
\hline 56 & -4.5 & -24.8 & n.d. & 0.92 & 183 \\
\hline 57 & -9.0 & n.d. & n.d. & 0.43 & 67.1 \\
\hline 58 & -2.1 & -8.1 & -29 & n.d. & n.d. \\
\hline 59 & n.d. & n.d. & n.d. & n.d. & n.d. \\
\hline 60 & -0.6 & -12.3 & -54 & 6.21 & 92.8 \\
\hline 61 & -0.1 & -19.5 & -136 & 6.49 & 224 \\
\hline 62 & -1.9 & -18.3 & n.d. & 2.38 & 12.8 \\
\hline 63 & -1.0 & -18.2 & n.d. & n.d. & n.d. \\
\hline 64 & n.d. & n.d. & n.d. & n.d. & n.d. \\
\hline 65 & 0.3 & -13.4 & n.d. & n.d. & n.d. \\
\hline 66 & -0.5 & -18.0 & -128 & 6.71 & 1070 \\
\hline 67 & -20.1 & -12.5 & -14 & 3.88 & 48.3 \\
\hline 68 & -2.5 & n.d. & n.d. & 0.85 & 5.9 \\
\hline 69 & -0.7 & n.d. & n.d. & 4.22 & 1.6 \\
\hline 70 & -1.2 & n.d. & n.d. & 4.98 & 25.8 \\
\hline 71 & -2.1 & n.d. & n.d. & n.d. & n.d. \\
\hline 72 & 0.4 & -16.1 & -101 & 4.62 & 139 \\
\hline
\end{tabular}

Table 2 (continued)

\begin{tabular}{|c|c|c|c|c|c|}
\hline N. & $\delta^{13} \mathrm{C}\left(\mathrm{CO}_{2}\right)$ & $\delta^{13} \mathrm{C}\left(\mathrm{CH}_{4}\right)$ & $\delta^{2} \mathrm{H}\left(\mathrm{CH}_{4}\right)$ & $\mathrm{R} / \mathrm{R}_{\mathrm{A}}$ & ${ }^{4} \mathrm{He} /{ }^{20} \mathrm{Ne}$ \\
\hline & $\%$ & $\%$ & $\% 0$ & & \\
\hline 73 & -2.7 & n.d. & n.d. & 3.22 & 8.7 \\
\hline 74 & -2.1 & n.d. & n.d. & 3.35 & 19.5 \\
\hline 75 & 0.3 & -34.8 & -174 & n.d. & n.d. \\
\hline 76 & -1.0 & n.d. & n.d. & n.d. & n.d. \\
\hline 77 & n.d. & n.d. & n.d. & n.d. & n.d. \\
\hline 78 & -1.9 & -26.1 & n.d. & 0.40 & 335 \\
\hline 79 & -1.9 & -26.3 & n.d. & n.d. & n.d. \\
\hline 80 & -3.1 & n.d. & n.d. & 1.04 & 2.9 \\
\hline 81 & -3.0 & n.d. & n.d. & 1.55 & 44.1 \\
\hline 82 & 6.0 & -44.6 & -263 & 0.72 & 0.74 \\
\hline 83 & -0.9 & -27.7 & -143 & 1.15 & 185 \\
\hline 84 & -5.8 & 34.7 & n.d. & 0.44 & 18.1 \\
\hline 85 & -12.3 & n.d. & n.d. & 0.51 & 96.4 \\
\hline 86 & 0.3 & n.d. & n.d. & 2.65 & 0.92 \\
\hline 87 & -2.3 & -12.0 & n.d. & n.d. & n.d. \\
\hline 88 & n.d. & -2.0 & -127 & 0.97 & 0.82 \\
\hline 89 & n.d. & -26.6 & -301 & 0.87 & 0.93 \\
\hline 90 & n.d. & -35.0 & -288 & 0.38 & 0.84 \\
\hline 91 & n.d. & -72.3 & -174 & n.d. & n.d. \\
\hline 92 & n.d. & -49.2 & -62 & 0.08 & 8.7 \\
\hline 93 & n.d. & -37.5 & -204 & 0.07 & 16.4 \\
\hline 94 & n.d. & -42.3 & -154 & 0.06 & 17.1 \\
\hline 95 & -1.7 & -22.0 & -108 & 5.66 & 536 \\
\hline 96 & -1.6 & -23.6 & -125 & 6.08 & 30.1 \\
\hline 97 & -2.7 & -23.3 & -121 & 5.78 & 242 \\
\hline 98 & -2.1 & -23.6 & -110 & 5.53 & 6.8 \\
\hline 99 & -2.1 & -23.3 & -130 & 5.91 & 29.1 \\
\hline 100 & -3.5 & 2.4 & 36 & 1.44 & 40.6 \\
\hline 101 & -0.8 & -16.1 & -75 & 3.67 & 81.5 \\
\hline
\end{tabular}

n.d. $=$ not determined

comprised between 1 and 15\%. Also gases from HH show a mix between a low mantle component (2-5\%) and a prevailing crustal source.

In the $\mathrm{CO}_{2} /{ }^{3} \mathrm{He}$ vs. $\delta^{13} \mathrm{C}-\mathrm{CO}_{2}$ binary diagram (Fig. $4 \mathrm{~b}$ ), the $\mathrm{CO}_{2}$ - and $\mathrm{N}_{2}$-dominated gases from VA and the $\mathrm{N}_{2}-\mathrm{CO}_{2}$ thermal emissions from $\mathrm{IH}$ mostly plot along the mixing line between the mantle and the limestone end-members, showing a low contribution from sedimentary organicrich sources. The $\mathrm{N}_{2}$-dominated gases of the cold manifestations from $\mathrm{IH}$ show $\mathrm{CO}_{2} /{ }^{3} \mathrm{He}$ ratios even lower than that of mantle gases, which are probably caused by relative $\mathrm{CO}_{2}$ loss due to its higher solubility in aquatic environments with respect to $\mathrm{He}$ (Fig. $4 \mathrm{~b}$ ). This process may explain the extremely low $\delta^{13} \mathrm{C}-\mathrm{CO}_{2}$ and $\mathrm{CO}_{2} /{ }^{3} \mathrm{He}$ values shown by sample 67 from the VA $\left(-20.1 \% 0\right.$ and $3.7 \times 10^{5}$, respectively), which was characterised by a relatively low concentration of $\mathrm{CO}_{2}$. Carbon dioxide loss may also be produced by calcite deposition at relatively high pH (Stefánsson et al., 2016, 2017). This process involves deprotonation of $\mathrm{CO}_{2}$ (aq) to $\mathrm{HCO}_{3}{ }^{-}$and $\mathrm{CO}_{3}{ }^{-2}$ and formation of calcite, resulting in a decrease of the $\delta^{13} \mathrm{C}$ values of the residual $\mathrm{CO}_{2}$. It should be noted that most thermal water samples are oversaturated in carbonate minerals and travertine depositions were recognized at some thermal areas (Kanellopoulos, 2012; Winkel et al., 2013; Kanellopoulos et al., 2017).

Hydrogen sulfide in fumarolic gases from active volcanoes is likely produced by thermochemical reduction of magmatic $\mathrm{SO}_{2}$ occurring within the hydrothermal reservoirs (Giggenbach, 1987) and, therefore, shows the highest concentrations along the VA (Fig. 3f) where most of the geothermal systems evidence an input of magmatic gases (Marini and Fiebig, 2005; Rizzo et al., 2015). Sedimentary sources of $\mathrm{H}_{2} \mathrm{~S}$ consist of i) alteration of sulfide minerals (Giggenbach, 1980; Chiodini, 1994), ii) microbial activity, and iii) thermochemical sulfate reduction (Machel et al., 1995; Worden and Smalley, 1996). The $\mathrm{H}_{2} \mathrm{~S}$ concentrations in the gas discharges of $\mathrm{EH}$ and $\mathrm{HH}$ are below detection limit with few exceptions, likely because this compound has a relatively high solubility in water and/or the lack of significant sources of S-bearing volatile compounds in the regions. Thus, whichever its origin, it may be 

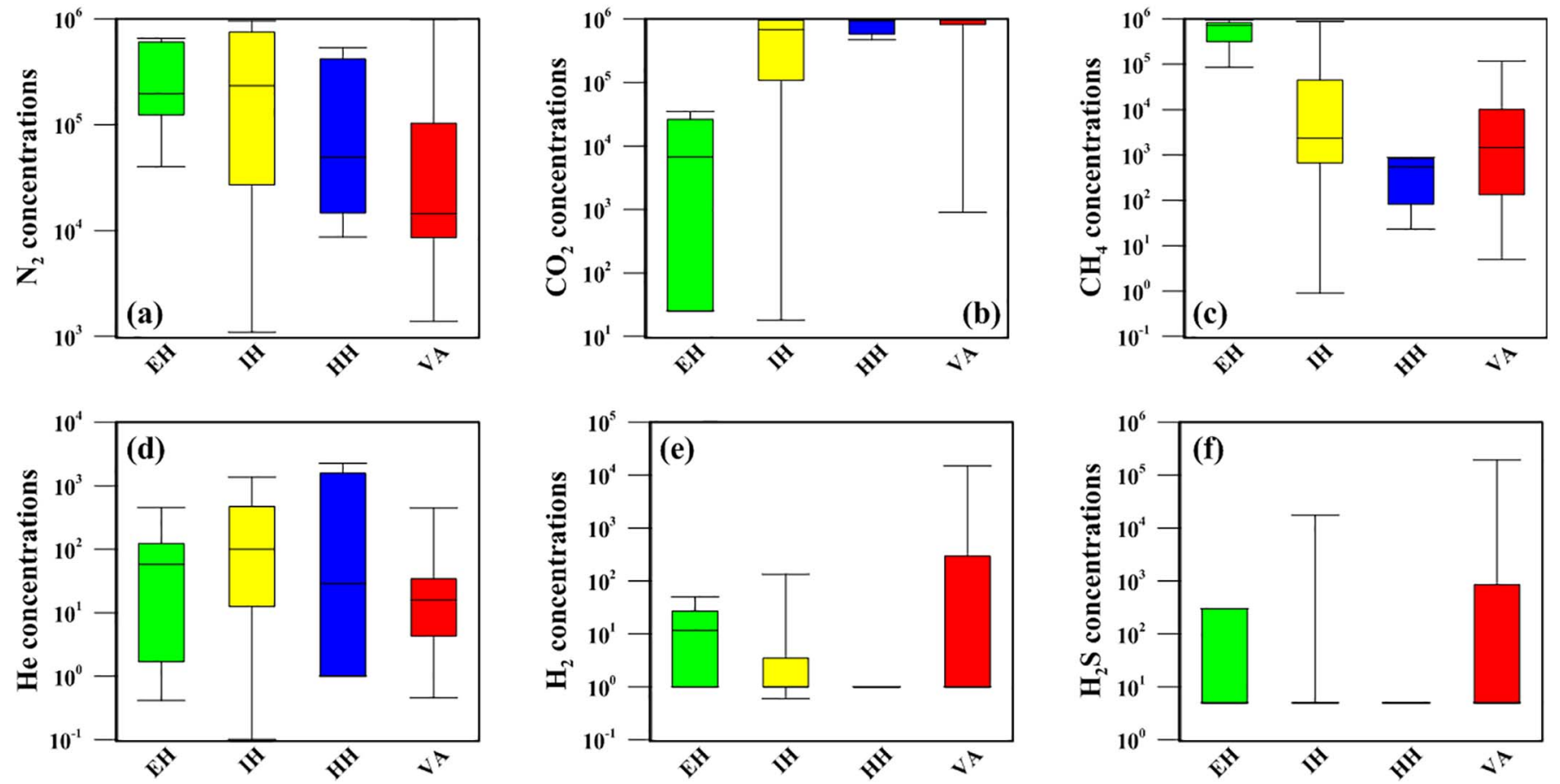

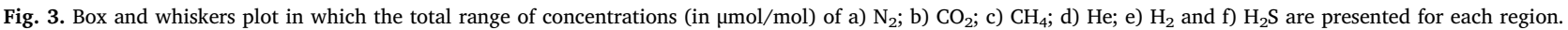

partially or totally dissolved and oxidized in cold shallow aquifers present along the uprising gas pathways (Minissale, 2004). Most gases from IH show also $\mathrm{H}_{2} \mathrm{~S}$ concentrations below detection limit, excepting samples $6,23,30,31$ and 36 where concentrations up to $17,400 \mu \mathrm{mol} /$ mol have been measured. It is worth noting that these samples were collected in two areas (Sperchios basin and Kassandra peninsula) corresponding to maxima on the heat flow map (Fig. 1c), suggesting the occurrence of medium enthalpy geothermal systems (Fytikas and Kolios, 1979).

The occurrence of relatively high concentrations of $\mathrm{H}_{2}$ (up to $14,800 \mu \mathrm{mol} / \mathrm{mol}$; Table 1 , Fig. 3e) in gases from the volcanic systems of Nisyros and Santorini is most likely related to water-rock interactions at elevated temperatures. It is well known that Fe(II)-bearing minerals react with water at temperatures $>300{ }^{\circ} \mathrm{C}$ generating $\mathrm{H}_{2}$ (e.g., Giggenbach, 1987; Seewald, 2001). Most of the samples collected at IH and $\mathrm{HH}$ have $\mathrm{H}_{2}$ below detection limit (Table 1). Concentrations of $\mathrm{H}_{2}$ up to $50 \mu \mathrm{mol} / \mathrm{mol}$, a gas often linked to hydrocarbon generation, are

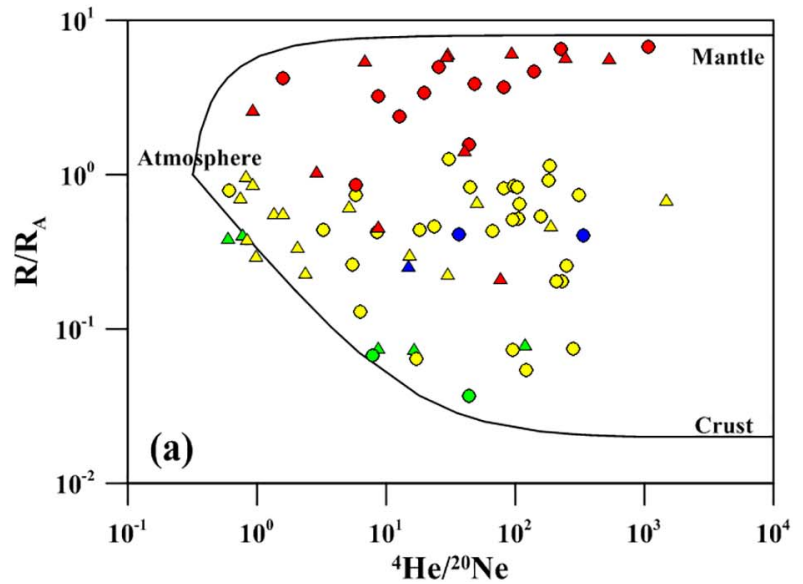

found in the EH gases related to hydrocarbon reservoirs of the area.

\subsection{An overview in the classification of hydrocarbons}

Taran and Giggenbach (2003) highlighted that for the description of hydrothermal hydrocarbon production two main mechanisms can be hypothesized. The first one deals with the biotic origin of methane, whereas the second one with its abiotic origin.

Gunter (1978), based on numerous observations, proposed that the nature and distribution of hydrocarbon species in hydrothermal vapours is more consistent with the thermal degradation of kerogen rather than inorganic production, a conclusion also supported by other researchers (e.g., Welhan, 1988; Darling, 1998; Mango, 2000; Taran and Giggenbach, 2003). More specifically, as indicated by Hunt (1996), biotic methane is produced either by microbial or thermogenic processes.

The second approach has to do with the formation of abiotic (or

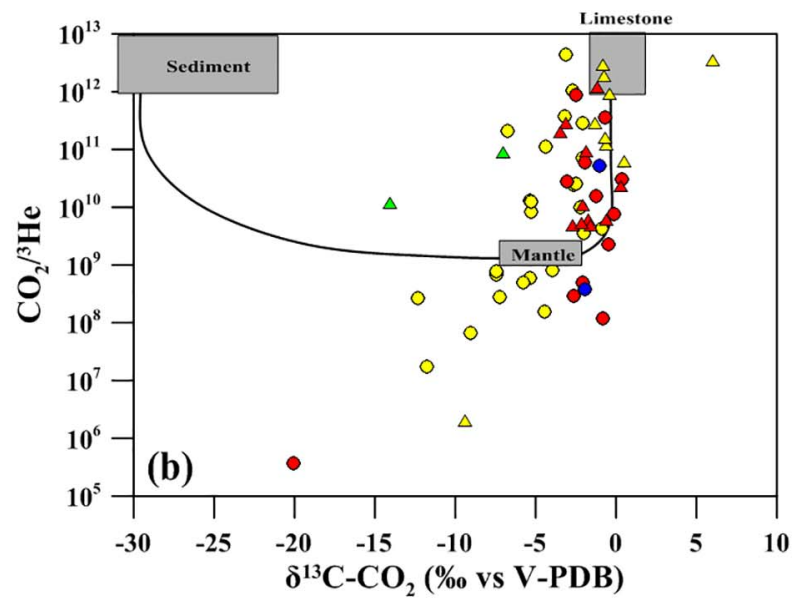

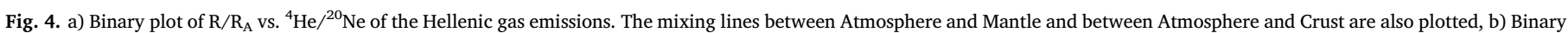

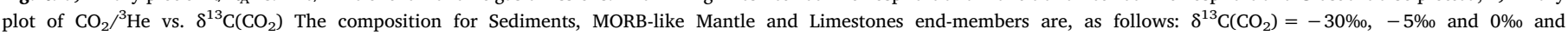
$\mathrm{CO}_{2} /{ }^{3} \mathrm{He}=1 \times 10^{13}, 2 \times 10^{9}$ and $1 \times 10^{13}$, respectively (Sano and Marty, 1995). Symbols as in Fig. 3 . 
abiogenic) methane by chemical reactions that do not require the presence of organic matter. These reactions include magmatic processes and gas-water-rock reactions (for example Fischer Tropsch type reactions (Fischer and Tropsch, 1923, 1926)) occurring over a wide range of temperatures (Etiope and Sherwood Lollar, 2013). These processes refer to the formation of organic compounds from a gaseous mixture of $\mathrm{CO}$ and $\mathrm{H}_{2}$ in high temperature and pressure surface-catalysed process, involving sequential reduction and polymerization of single-carbon units (McCollom and Seewald, 2007; McCollom, 2013; Konn et al., 2015), (see Reaction (1)). From this process, it is noticeable that trace amounts of abiotic hydrocarbons can occur in volcanic and geothermal fluids. Moreover, considerable amounts of methane, reaching concentrations of 80-90 vol\%, have also been discovered in an increasing number of sites in Precambrian crystalline shields, in serpentinized ultramafic rocks along mid-ocean ridges and in land-based ophiolites, peridotite massifs, and igneous intrusions (Etiope and Sherwood Lollar, 2013).

Considering these mechanisms, generally, although not exclusively, the origin of $\mathrm{CH}_{4}$ can be investigated using the classification diagrams proposed by Bernard and Schoell (Bernard et al., 1978; Schoell, 1980, 1988; Etiope and Schoell, 2014, Fig. 6a, b). Bernard et al. (1978) introduced correlation between $\mathrm{CH}_{4} /\left(\mathrm{C}_{2} \mathrm{H}_{6}+\mathrm{C}_{3} \mathrm{H}_{8}\right)$ concentration ratios and the $\delta^{13} \mathrm{C}-\mathrm{CH}_{4}$ ratios to qualitatively distinguish $\mathrm{CH}_{4}$ derived from microbial and thermogenic sources. Furthermore, other researchers (Whiticar et al., 1986; Schoell, 1988; Burke et al., 1988, 1988; Whiticar, 1999a, 1999b; Hornibrook et al., 1997; Chanton et al., 2005) hypothesized that the production of $\mathrm{CH}_{4}$ is associated with inverse or antipathetic shifts in $\delta^{2} \mathrm{H}$ and $\delta^{13} \mathrm{C}_{\text {of }} \mathrm{CH}_{4}$, in which fermentation of acetate will result in $\mathrm{CH}_{4}$ enriched in ${ }^{13} \mathrm{C}$ and depleted in ${ }^{2} \mathrm{H}$, something relative to $\mathrm{CH}_{4}$ produced via the $\mathrm{CO}_{2}$ reduction pathway. In those diagrams (Fig. 6a, b), thermogenic $\mathrm{CH}_{4}$ has been reported to exhibit $\delta^{13} \mathrm{C}_{-} \mathrm{CH}_{4}$ values ranging from -50 to $-30 \%$ and $\delta^{2} \mathrm{H}-\mathrm{CH}_{4}$ values $\leq 150 \%$ (e.g., Schoell, 1980; Whiticar, 1999a, 1999b; Bradley and Summons, 2010). Microbial $\mathrm{CH}_{4}$ usually has $\delta^{13} \mathrm{C}_{-} \mathrm{CH}_{4}$ values $\leq 50 \%$ (e.g. Whiticar, 1999a, 1999b; McCollom and Seewald, 2007) and can be produced either by i) methyl-type fermentation or by ii) carbonate reduction $\left({ }^{13} \mathrm{C}\right.$-depleted and ${ }^{2} \mathrm{H}$-enriched $\mathrm{CH}_{4}$ with respect to the methyltype fermentation) (Whiticar et al., 1986; Whiticar, 1999a, 1999b). $\mathrm{CO}_{2}$-reduction and acetate fermentation pathways may be distinguished also on the basis of the carbon isotope fractionation factor $\left(\alpha_{\mathrm{C}}\right)$ between coexisting $\mathrm{CO}_{2}$ and $\mathrm{CH}_{4}$ (Whiticar et al., 1986).

$\alpha_{\mathrm{C}}=\left(\delta^{13} \mathrm{C}-\mathrm{CO}_{2}+1000\right) /\left(\delta^{13} \mathrm{C}-\mathrm{CH}_{4}+1000\right)$

The isotope fractionation of the carbon dioxide reduction is generally between $\alpha_{C}=1.055$ and 1.09 , in contrast to acetate- type fermentation which is usually between $\alpha_{\mathrm{C}}=1.04$ and 1.055 .

Gases produced by the decay of organic matter at $T>150{ }^{\circ} \mathrm{C}$ (thermogenic gases) are commonly characterised by $\mathrm{CH}_{4}$ / $\left(\mathrm{C}_{2} \mathrm{H}_{6}+\mathrm{C}_{3} \mathrm{H}_{8}\right)$ concentration ratios (known as the "Bernard parameter") $<100$, with the exception of coal gases (Whiticar, 1999a, 1999b) and shale gases (Tilley and Muehlenbachs, 2013). Higher ratios ( $>1000)$ are expected when hydrocarbon production derives exclusively from microbial activity (Whiticar and Suess, 1990; Jenden et al., 1993). However, many processes can play a fundamental role for its origin, such as isotopic fractionation by diffusion (Prinzhofer and Battani, 2003), secondary methanogenesis and anaerobic biodegradation (Dimitrakopoulos and Muehlenbachs, 1987; Pallasser, 2000; Etiope et al., 2009). Methanotrophic bacteria, for instance, may oxidize biogenic $\mathrm{CH}_{4}$, causing a shift toward less negative isotopic values of the residual gas (Coleman et al., 1981).

$\mathrm{CH}_{4}$ of abiogenic origin, e.g. discharged from high-temperature hydrothermal fluids venting from sediment-free ultramafic systems, is thought to be characterised by $\delta^{2} \mathrm{H}-\mathrm{CH}_{4}$ values higher than $-150 \%$ (Welhan and Craig, 1983; Proskurowski et al., 2006, 2008; McCollom and Seewald, 2007; Bradley and Summons, 2010). Similarly, the $\delta^{13} \mathrm{C}$ $\mathrm{CH}_{4}$ values of $\mathrm{CH}_{4}$ produced from water-rock reactions, such as hydration of ultramafic rocks, may vary in a wide range because it depends on the inorganic carbon source and the magnitude of carbon isotope fractionation.

Another possible source of abiogenic $\mathrm{CH}_{4}$ is the serpentinization process of ultramafic rocks within ophiolitic sequences. Such processes produce significant quantities of $\mathrm{H}_{2}$ even at temperatures below $100{ }^{\circ} \mathrm{C}$ (Miller et al., 2017) mainly through the following reactions:

$2(\mathrm{Mg}, \mathrm{Fe})_{2} \mathrm{SiO}_{4}+3 \mathrm{H}_{2} \mathrm{O} \rightarrow(\mathrm{Mg}, \mathrm{Fe})_{3} \mathrm{Si}_{2} \mathrm{O}_{5}(\mathrm{OH})_{4}+(\mathrm{Mg}, \mathrm{Fe})(\mathrm{OH})_{2}$ olivine serpentine brucite

$\mathrm{Fe}_{3} \mathrm{Si}_{2} \mathrm{O}_{5}(\mathrm{OH})_{4} \quad+\mathrm{H}_{2} \mathrm{O} \rightarrow \mathrm{Fe}_{3} \mathrm{O}_{4}+2 \mathrm{SiO}_{2}+\mathrm{H}_{2}$ serpentine (greenalite) magnetite silica

The produced $\mathrm{H}_{2}$ in turn may be transformed in $\mathrm{CH}_{4}$ reacting with carbon oxides ( $\mathrm{CO}, \mathrm{CO}_{2}$ or simple organic acids). Such reactions can be mediated either microbially or inorganically (Fischer-Tropsch type reactions - Etiope and Sherwood Lollar, 2013). The latter are favoured at high temperatures but experiments show that they can proceed even at ambient temperature in the presence of a suitable catalyst (Etiope and Ionescu, 2015). Apart from significant $\mathrm{H}_{2}$ and $\mathrm{CH}_{4}$ gas production, the serpentinization processes lead to the formation of hyperalkline groundwaters (Barnes and O'Neil, 1969; Bruni et al., 2002).

Besides few exceptions (Hinrichs et al., 2006), Bernard and Schoell diagrams (Bernard et al., 1978; Schoell, 1980, 1988; Etiope and Schoell, 2014, Fig. 6a, b) work fairly well for the classification of $\mathrm{CH}_{4}$ from thermogenic and microbial sources, however they are of limited use considering the identification of abiogenic $\mathrm{CH}_{4}$. The reason is that there seems to be a considerable overlap of fields characteristic for thermogenic $\mathrm{CH}_{4}$, thermogenic $\mathrm{CH}_{4}$ affected by secondary oxidation and "abiogenic" $\mathrm{CH}_{4}$. Furthermore, some studies evidenced that in carbon limited conditions the Carbon isotopic composition of $\mathrm{CH}_{4}$ deriving from microbial activity could reach values as high as those generally attributed to abiogenic origin (Kietäväinen and Purmako, 2015).

Recently, new proxies such as clumped isotopes, capable of determining the isotopologues of $\mathrm{CH}_{4}$ molecules (Stolper et al., 2014; Wang et al., 2015), have been developed. Potential applications of this method include determination of $\mathrm{CH}_{4}$ formation temperature $\left(\mathrm{CH}_{4}\right.$ thermometry), and detection of kinetic isotope fractionation, both of which might be used in separating biotic from abiotic $\mathrm{CH}_{4}$ (Stolper et al., 2014; Wang et al., 2015). More applications are expected when this method comes more widely attainable.

\subsection{Origin of hydrocarbon compounds in Greece}

Taking into consideration the geo-lithological division of the study areas and the dominant gas species, it is noticeable that the manifestations found on the western part of Greece display higher $\mathrm{CH}_{4}$ concentrations (Fig. 3c) with respect to those found in the eastern part where $\mathrm{CO}_{2}$ is the prevailing gas species (Figs. 1a, 2a and 3b). In particular, EH are characterised by widespread and thick sedimentary formations mainly originating from the erosion of elder rocks from $\mathrm{IH}$ and $\mathrm{HH}$. This geological/petrological regime makes the stratigraphic sequences of $\mathrm{EH}$ richer in solid organic substances, as also supported by the hydrocarbon deposits occurring in this area (Palacas et al., 1986). On the other hand, IH and $\mathrm{HH}$ are characterised by more "mature" formations (Cavazza et al., 2014), mainly intrusive and metamorphic, and by significant erosion causing a substantial reduction of the crustal thickness before the post-orogenic extension. These formations contain much less organic matter than EH. Therefore, as also seen in Fig. 6a and b, the occurrence of biogenic $\mathrm{CH}_{4}$ in these areas is likely related to the presence of a post-orogenic sedimentary cover.

The most used parameters to discriminate the genetic processes of $\mathrm{CH}_{4}$ are the C- and H-isotopic compositions of $\mathrm{CH}_{4}$ (Schoell, 1980; Etiope and Schoell, 2014) and the $\mathrm{CH}_{4} /\left(\mathrm{C}_{2} \mathrm{H}_{6}+\mathrm{C}_{3} \mathrm{H}_{8}\right)$ ratio (Bernard et al., 1978). Of course, it should be considered that secondary 
Table 3

Concentrations of $\mathrm{C}_{2}-\mathrm{C}_{6}$ hydrocarbons.

\begin{tabular}{|c|c|c|c|c|c|c|c|c|c|c|}
\hline $\mathrm{N}$. & $\mathrm{C}_{2} \mathrm{H}_{6}$ & $\mathrm{C}_{3} \mathrm{H}_{8}$ & $\mathrm{C}_{3} \mathrm{H}_{6}$ & $i-\mathrm{C}_{4} \mathrm{H}_{10}$ & $n-\mathrm{C}_{4} \mathrm{H}_{10}$ & $i-\mathrm{C}_{4} \mathrm{H}_{8}$ & $i-\mathrm{C}_{5} \mathrm{H}_{12}$ & $n-\mathrm{C}_{5} \mathrm{H}_{12}$ & $\mathrm{C}_{6} \mathrm{H}_{6}$ & $\mathrm{CH}_{4} /\left(\mathrm{C}_{2} \mathrm{H}_{6}+\mathrm{C}_{3} \mathrm{H}_{8}\right)$ \\
\hline & $\mu \mathrm{mol} / \mathrm{mol}$ & $\mu \mathrm{mol} / \mathrm{mol}$ & $\mu \mathrm{mol} / \mathrm{mol}$ & $\mu \mathrm{mol} / \mathrm{mol}$ & $\mu \mathrm{mol} / \mathrm{mol}$ & $\mu \mathrm{mol} / \mathrm{mol}$ & $\mu \mathrm{mol} / \mathrm{mol}$ & $\mu \mathrm{mol} / \mathrm{mol}$ & $\mu \mathrm{mol} / \mathrm{mol}$ & \\
\hline 1 & 112 & 2.8 & b.d.l. & 0.12 & b.d.l. & b.d.l. & b.d.l. & b.d.l. & b.d.l. & 7667 \\
\hline 2 & 21 & 3.7 & 0.018 & 2.3 & 2.1 & 3.7 & 0.45 & 0.45 & 4.9 & 45 \\
\hline 3 & 15 & 3.6 & 0.02 & 1.8 & 2.1 & 2.6 & 0.42 & 0.51 & 5.6 & 34 \\
\hline 4 & 13 & 2.8 & 0.03 & 2.6 & 1.2 & 1.5 & 0.55 & 0.33 & 2.2 & 64 \\
\hline 5 & 11 & 2.2 & 0.04 & 2.1 & 1.5 & 1.4 & 0.48 & 0.25 & 1.8 & 65 \\
\hline 6 & 5.1 & 1.3 & b.d.l. & 0.45 & 1.1 & 0.68 & 0.33 & 0.21 & 0.56 & 327 \\
\hline 7 & 8.7 & 2.2 & b.d.l. & 0.85 & 0.71 & 1.3 & 0.36 & 0.31 & 0.66 & 308 \\
\hline 8 & 13 & 3.6 & 0.01 & 1.4 & 1.7 & 1.5 & 0.62 & 0.33 & 0.98 & 245 \\
\hline 9 & 3.1 & 0.85 & b.d.l. & 0.33 & 0.75 & 0.55 & 0.69 & 0.25 & 0.44 & 276 \\
\hline 10 & 1.1 & 0.08 & b.d.l. & b.d.l. & b.d.l. & 0.51 & b.d.l. & b.d.l. & 0.54 & 256 \\
\hline 11 & 1.1 & 0.29 & b.d.l. & 0.12 & 0.17 & 0.22 & 0.09 & 0.05 & 0.28 & 19 \\
\hline 12 & 8.6 & 2.3 & 0.03 & 0.62 & 1.3 & 0.95 & 0.26 & 0.39 & 5.4 & 110 \\
\hline 13 & 95 & 11 & b.d.l. & b.d.l. & b.d.l. & b.d.l. & b.d.l. & b.d.l. & b.d.l. & 6645 \\
\hline 14 & n.d. & n.d. & n.d. & n.d. & n.d. & n.d. & n.d. & n.d. & n.d. & n.c. \\
\hline 15 & 2.1 & b.d.l. & b.d.l. & b.d.l. & b.d.l. & b.d.l. & b.d.l. & b.d.l. & b.d.l. & 4810 \\
\hline 16 & 15 & 3.1 & 0.06 & 2.5 & 3.1 & 5.5 & 1.3 & 0.66 & 5.1 & 101 \\
\hline 17 & 13 & 3.3 & 0.08 & 2.3 & 2.6 & 4.4 & 1.5 & 0.71 & 4.5 & 110 \\
\hline 18 & 3.8 & 0.46 & b.d.l. & 0.15 & 0.16 & 0.11 & 0.08 & 0.06 & 0.71 & 244 \\
\hline 19 & 2.3 & 0.51 & b.d.l. & 0.15 & 0.26 & 0.75 & 0.08 & 0.11 & 0.85 & 19 \\
\hline 20 & 1.5 & 0.23 & b.d.l. & 0.11 & 0.22 & 0.54 & 0.05 & 0.08 & 0.77 & 18 \\
\hline 21 & 10 & 2.1 & 0.02 & 1.1 & 0.65 & 0.85 & 0.44 & 0.26 & 0.77 & 76 \\
\hline 22 & 6.1 & 1.5 & 0.02 & 1.1 & 0.66 & 0.74 & 0.21 & 0.22 & 1.2 & 62 \\
\hline 23 & 1.5 & 0.11 & b.d.l. & b.d.l. & 0.06 & b.d.l. & b.d.l. & b.d.l. & b.d.l. & 6832 \\
\hline 24 & 3.4 & 0.56 & 0.02 & 0.41 & 0.36 & 0.41 & 0.56 & 0.15 & 0.74 & 54 \\
\hline 25 & 123 & 3.5 & b.d.l. & 0.15 & b.d.l. & b.d.l. & b.d.l. & b.d.1. & b.d.l. & 7221 \\
\hline 26 & 2960 & 278 & 0.21 & 126 & 178 & 155 & 26 & 35 & 215 & 201 \\
\hline 27 & 321 & 8.5 & b.d.l. & b.d.l. & b.d.l. & b.d.l. & b.d.l. & b.d.1. & b.d.l. & 2579 \\
\hline 28 & 11 & 3.3 & b.d.l. & 1.2 & 1.3 & 1.6 & 0.56 & 0.61 & 0.98 & 400 \\
\hline 29 & 13 & 0.51 & b.d.l. & b.d.l. & b.d.l. & b.d.l. & b.d.l. & b.d.l. & b.d.l. & 5751 \\
\hline 30 & 13 & 3.1 & b.d.l. & 2.5 & 1.4 & 1.5 & 0.39 & 0.33 & 0.91 & 277 \\
\hline 31 & 6.5 & 0.85 & b.d.l. & 0.41 & 0.85 & 0.76 & 0.05 & 0.13 & 1.4 & 790 \\
\hline 32 & 4.3 & 0.85 & 0.02 & 0.41 & 0.69 & 0.64 & 0.15 & 0.22 & 2.1 & 155 \\
\hline 33 & 2.4 & 0.39 & b.d.l. & 0.15 & 0.21 & 0.26 & 0.05 & 0.06 & 0.65 & 89 \\
\hline 34 & 2.1 & 0.33 & b.d.l. & 0.11 & 0.25 & 0.29 & 0.11 & 0.12 & 0.58 & 357 \\
\hline 35 & 6.6 & 1.3 & 0.04 & 0.55 & 1.1 & 0.88 & 0.12 & 0.26 & 3.7 & 87 \\
\hline 36 & 6.7 & 1.6 & b.d.l. & 0.55 & 0.69 & 1.3 & 0.25 & 0.36 & 2.4 & 63 \\
\hline 37 & 12 & 2.5 & 0.04 & 1.5 & 2.1 & 3.6 & 1.1 & 0.66 & 1.8 & 186 \\
\hline 38 & 15 & 2.9 & 0.03 & 1.1 & 2.6 & 3.1 & 0.74 & 0.51 & 2.2 & 131 \\
\hline 39 & 3.1 & 0.15 & b.d.l. & b.d.l. & b.d.l. & b.d.l. & b.d.l. & b.d.l. & b.d.l. & 1908 \\
\hline 40 & 7.7 & 2.1 & 0.03 & 1.2 & 0.77 & 1.1 & 0.25 & 0.26 & 1.5 & 49 \\
\hline 41 & 8.9 & 1.8 & 0.006 & 1.1 & 0.85 & 1.3 & 0.21 & 0.21 & 1.7 & 20 \\
\hline 42 & b.d.l. & b.d.l. & b.d.l. & b.d.l. & b.d.l. & b.d.l. & b.d.l. & b.d.1. & b.d.l. & n.c. \\
\hline 43 & b.d.l. & b.d.l. & b.d.l. & b.d.l. & b.d.l. & b.d.l. & b.d.l. & b.d.l. & b.d.l. & n.c. \\
\hline 44 & 19 & 3.9 & 0.11 & 2.4 & 2.8 & 4.9 & 1.8 & 0.75 & 4.1 & 114 \\
\hline 45 & b.d.l. & b.d.l. & b.d.l. & b.d.l. & b.d.l. & b.d.l. & b.d.l. & b.d.l. & b.d.l. & n.c. \\
\hline 46 & 0.08 & b.d.l. & b.d.l. & b.d.l. & b.d.l. & b.d.l. & b.d.l. & b.d.1. & b.d.l. & 25 \\
\hline 47 & 61 & 3.9 & b.d.l. & b.d.l. & b.d.l. & b.d.l. & b.d.l. & b.d.l. & b.d.l. & 6712 \\
\hline 48 & 3.6 & 0.66 & b.d.l. & 0.65 & 0.78 & 1.2 & 0.15 & 0.24 & 1.8 & 5.4 \\
\hline 49 & 4.3 & 0.71 & b.d.l. & 0.78 & 0.85 & 1.6 & 0.21 & 0.26 & 1.9 & 16 \\
\hline 50 & 85 & 3.1 & b.d.l. & b.d.l. & b.d.l. & b.d.l. & b.d.l. & b.d.l. & b.d.l. & 8675 \\
\hline 51 & 5.3 & 1.3 & 0.008 & 0.31 & 0.39 & 0.75 & 0.08 & 0.07 & 0.56 & 101 \\
\hline 52 & 2.3 & 0.15 & 0.02 & 0.11 & 0.14 & 0.25 & 0.08 & 0.06 & 0.58 & 287 \\
\hline 53 & 3.6 & b.d.l. & b.d.l. & b.d.l. & b.d.l. & b.d.l. & b.d.l. & b.d.l. & b.d.l. & 9111 \\
\hline 54 & 2.3 & b.d.l. & b.d.l. & b.d.l. & b.d.l. & b.d.l. & b.d.l. & b.d.l. & b.d.l. & 8913 \\
\hline 55 & 12 & 3.3 & 0.02 & 1.4 & 2.1 & 3.3 & 0.55 & 1.2 & 2.3 & 14 \\
\hline 56 & b.d.l. & b.d.l. & b.d.l. & b.d.l. & b.d.l. & b.d.l. & b.d.l. & b.d.l. & b.d.l. & n.c. \\
\hline 57 & 0.51 & 0.18 & b.d.l. & 0.05 & b.d.l. & 0.11 & b.d.l. & b.d.l. & 0.34 & 14 \\
\hline 58 & 22 & 5.6 & 0.008 & 2.2 & 2.9 & 3.7 & 1.1 & 1.2 & 7.1 & 168 \\
\hline 59 & 9.5 & 2.1 & 0.007 & 1.3 & 1.1 & 1.9 & 0.26 & 0.31 & 2.6 & 71 \\
\hline 60 & 12 & 0.89 & b.d.l. & b.d.l. & b.d.l. & b.d.l. & b.d.l. & b.d.l. & b.d.l. & 1853 \\
\hline 61 & 1.6 & 0.15 & b.d.l. & b.d.l. & 0.12 & b.d.l. & b.d.l. & b.d.l. & 0.08 & 6914 \\
\hline 62 & 7.3 & 0.33 & b.d.l. & 0.11 & 0.21 & 0.25 & 0.08 & 0.05 & 0.15 & 389 \\
\hline 63 & 0.15 & b.d.l. & b.d.l. & b.d.l. & b.d.l. & b.d.l. & b.d.l. & b.d.l. & b.d.l. & 16,000 \\
\hline 64 & 8.1 & 0.48 & b.d.l. & 0.16 & 0.25 & 0.33 & 0.12 & 0.11 & 0.21 & 366 \\
\hline 65 & 4.2 & 0.26 & b.d.l. & 0.13 & 0.21 & 0.25 & 0.05 & b.d.l. & 0.11 & 4709 \\
\hline 66 & 4.3 & 0.85 & b.d.l. & 0.54 & 0.15 & 0.65 & 0.15 & 0.22 & 1.3 & 4485 \\
\hline 67 & 7.5 & 1.2 & 0.04 & 0.58 & 0.74 & 0.65 & 0.06 & 0.11 & 1.2 & 718 \\
\hline 68 & 0.45 & 0.13 & b.d.l. & b.d.l. & b.d.l. & 0.15 & b.d.l. & b.d.l. & 0.23 & 41 \\
\hline 69 & b.d.l. & b.d.l. & b.d.l. & b.d.l. & b.d.l. & b.d.l. & b.d.l. & b.d.l. & b.d.l. & n.c. \\
\hline 70 & 0.99 & 0.23 & b.d.l. & 0.08 & 0.11 & 0.05 & b.d.l. & b.d.l. & 0.39 & 56 \\
\hline 71 & 5.1 & 1.8 & 0.02 & 1.7 & 0.56 & 0.75 & 0.35 & 0.15 & 1.4 & 76 \\
\hline 72 & 11 & 0.33 & b.d.l. & b.d.l. & b.d.l. & b.d.l. & b.d.l. & b.d.l. & b.d.l. & $\begin{array}{l}1854 \\
\text { (continued on next page) }\end{array}$ \\
\hline
\end{tabular}


Table 3 (continued)

\begin{tabular}{|c|c|c|c|c|c|c|c|c|c|c|}
\hline N. & $\mathrm{C}_{2} \mathrm{H}_{6}$ & $\mathrm{C}_{3} \mathrm{H}_{8}$ & $\mathrm{C}_{3} \mathrm{H}_{6}$ & $i-\mathrm{C}_{4} \mathrm{H}_{10}$ & $n-\mathrm{C}_{4} \mathrm{H}_{10}$ & $i-\mathrm{C}_{4} \mathrm{H}_{8}$ & $i-\mathrm{C}_{5} \mathrm{H}_{12}$ & $n-\mathrm{C}_{5} \mathrm{H}_{12}$ & $\mathrm{C}_{6} \mathrm{H}_{6}$ & $\mathrm{CH}_{4} /\left(\mathrm{C}_{2} \mathrm{H}_{6}+\mathrm{C}_{3} \mathrm{H}_{8}\right)$ \\
\hline & $\mu \mathrm{mol} / \mathrm{mol}$ & $\mu \mathrm{mol} / \mathrm{mol}$ & $\mu \mathrm{mol} / \mathrm{mol}$ & $\mu \mathrm{mol} / \mathrm{mol}$ & $\mu \mathrm{mol} / \mathrm{mol}$ & $\mu \mathrm{mol} / \mathrm{mol}$ & $\mu \mathrm{mol} / \mathrm{mol}$ & $\mu \mathrm{mol} / \mathrm{mol}$ & $\mu \mathrm{mol} / \mathrm{mol}$ & \\
\hline 73 & 3.3 & 0.59 & 0.02 & 0.24 & 0.25 & 0.33 & 0.21 & 0.15 & 0.47 & 34 \\
\hline 74 & 7.6 & 2.5 & 0.08 & 1.1 & 0.95 & 1.6 & 0.78 & 1.1 & 2.9 & 14 \\
\hline 75 & 11 & 3.9 & 0.03 & 1.3 & 1.5 & 2.8 & 0.43 & 1.1 & 2.5 & 26 \\
\hline 76 & 4.6 & 0.91 & 0.08 & 0.45 & 0.45 & 0.69 & 0.11 & 0.25 & 2.6 & 137 \\
\hline 77 & 11 & 3.3 & 0.009 & 1.6 & 1.9 & 2.2 & 0.58 & 0.91 & 4.4 & 7.1 \\
\hline 78 & 6.2 & 1.5 & 0.008 & 1.6 & 1.8 & 2.3 & 0.66 & 0.91 & 2.1 & 110 \\
\hline 79 & 7.6 & 1.8 & 0.009 & 1.5 & 2 & 2.6 & 1.2 & 1.1 & 2.5 & 94 \\
\hline 80 & 4.3 & 1.1 & 0.006 & 0.23 & 0.36 & 0.81 & 0.11 & 0.13 & 1.9 & 9.4 \\
\hline 81 & 3.3 & 0.65 & 0.01 & 0.15 & 0.33 & 0.77 & 0.15 & 0.19 & 2.2 & 70 \\
\hline 82 & 60 & 3.9 & b.d.l. & b.d.l. & b.d.l. & b.d.l. & b.d.l. & b.d.l. & b.d.l. & 9013 \\
\hline 83 & 13 & 0.26 & b.d.l. & b.d.l. & b.d.l. & b.d.l. & b.d.l. & b.d.l. & b.d.l. & 7157 \\
\hline 84 & 7.8 & 3.1 & 0.05 & 0.56 & 0.71 & 0.56 & 0.54 & 0.23 & 1.4 & 76 \\
\hline 85 & b.d.l. & b.d.l. & b.d.l. & b.d.l. & b.d.l. & b.d.l. & b.d.l. & b.d.l. & b.d.l. & n.c. \\
\hline 86 & 5.6 & 1.1 & 0.15 & 0.26 & 0.54 & 0.78 & 0.31 & 0.11 & 2.3 & 39 \\
\hline 87 & 10 & 1.9 & 0.06 & 1.6 & 1.3 & 1.1 & 0.36 & 0.25 & 1.4 & 109 \\
\hline 88 & n.d. & n.d. & n.d. & n.d. & n.d. & n.d. & n.d. & n.d. & n.d. & n.c. \\
\hline 89 & 16.8 & b.d.l. & b.d.l. & b.d.l. & b.d.l. & b.d.l. & b.d.l. & b.d.l. & b.d.l. & 12,072 \\
\hline 90 & n.d. & n.d. & n.d. & n.d. & n.d. & n.d. & n.d. & n.d. & n.d. & n.c. \\
\hline 91 & 3.3 & 0.59 & 0.02 & 0.24 & 0.25 & 0.33 & 0.21 & 0.15 & 0.47 & 93,213 \\
\hline 92 & 120 & b.d.l. & b.d.l. & b.d.l. & b.d.l. & b.d.l. & b.d.l. & b.d.l. & b.d.l. & 2630 \\
\hline 93 & n.d. & n.d. & n.d. & n.d. & n.d. & n.d. & n.d. & n.d. & n.d. & n.c. \\
\hline 94 & n.d. & n.d. & n.d. & n.d. & n.d. & n.d. & n.d. & n.d. & n.d. & n.c. \\
\hline 95 & 0.9 & 0.18 & b.d.l. & b.d.l. & b.d.l. & b.d.l. & b.d.l. & b.d.l. & b.d.l. & 2331 \\
\hline 96 & n.d. & n.d. & n.d. & n.d. & n.d. & n.d. & n.d. & n.d. & n.d. & n.c. \\
\hline 97 & 5.51 & 1.1 & b.d.l. & b.d.l. & b.d.l. & b.d.l. & b.d.l. & b.d.l. & b.d.l. & 220 \\
\hline 98 & 4.48 & 0.75 & b.d.l. & b.d.l. & b.d.l. & b.d.l. & b.d.l. & b.d.l. & b.d.l. & 831 \\
\hline 99 & n.d. & n.d. & n.d. & n.d. & n.d. & n.d. & n.d. & n.d. & n.d. & n.c. \\
\hline 100 & n.d. & n.d. & n.d. & n.d. & n.d. & n.d. & n.d. & n.d. & n.d. & n.c. \\
\hline 101 & 23 & b.d.l. & b.d.l. & b.d.l. & b.d.l. & b.d.l. & b.d.l. & b.d.l. & b.d.l. & 5078 \\
\hline
\end{tabular}

b.d.l. = below detection limit; n.d. $=$ not determined; n.c. $=$ not calculated.

processes (e.g. mixing, inorganic or microbially-driven oxidation) affecting $\mathrm{CH}_{4}$ and light hydrocarbons might change the chemical and isotopic composition of these compounds masking, at least partially, their primary origin (Coleman et al., 1981; Kiyosu and Imaizumi, 1996; Kinnaman et al., 2007). High $\mathrm{CH}_{4} /\left(\mathrm{C}_{2} \mathrm{H}_{6}+\mathrm{C}_{3} \mathrm{H}_{8}\right)$ concentration ratios ( $>1000$ ) are generally limited to the "microbial" samples of $\mathrm{EH}$ and to many samples from IH, as well as for some samples of VA (Gyali and Kos islands) (Fig. 6a, Table 3). For the remaining samples, the ratio shows lower values, indicating either a preferential loss of $\mathrm{CH}_{4}$ due to microbial oxidation or the production of significant quantities of lowmolecular-weight hydrocarbons through thermal degradation of organic matter. More specifically, in both Bernard and modified Schoell diagrams (Fig. 6a, b), the $\mathrm{CH}_{4}$-dominated gases from $\mathrm{EH}$ plot in the biogenic fields. In particular, samples of the Gavrovo-Tripolis zone have a clear microbial origin with low $\delta^{13} \mathrm{C}$ but relatively high $\delta^{2} \mathrm{H}$ values of $\mathrm{CH}_{4}$ that point to a microbial carbonate reduction, whereas those from the Ionian zone seem to be of thermogenic origin (Fig. 6a, b). Some gas samples from the Ionian and of Pindos zones (EH) are intermediate between the thermogenic and microbially-derived group, (Fig. 6b). Such mixing pattern is also confirmed by the $\mathrm{CH}_{4} /\left(\mathrm{C}_{2} \mathrm{H}_{6}+\mathrm{C}_{3} \mathrm{H}_{8}\right)$ ratio measured in gases collected in petroleum exploration wells of the Katakolo hydrocarbon field (NW Peloponnesus), which decreases from pure microbial-type values $(1000-12,500)$ at shallower levels down to low values (3.27-24.4) at deeper levels (2000-2500 m depth) where temperatures for thermogenic gas generation are reached (Kamberis et al., 2000).

Samples ascribable to a microbial origin on the basis of their $\delta^{13} \mathrm{C}$ and $\delta^{2} \mathrm{H}$ values of $\mathrm{CH}_{4}$ display $\alpha_{\mathrm{C}}$ values (Fig. 5a) compatible with the $\mathrm{CO}_{2}$-reduction origin at least for the samples in which both $\delta^{13} \mathrm{C}^{-} \mathrm{CO}_{2}$ and $\delta^{13} \mathrm{C}^{-\mathrm{CH}_{4}}$ are available (samples 1, 13,25, 50 and 82).

Furthermore, some of the $\mathrm{N}_{2}-\mathrm{CO}_{2}$ dominated samples collected from the thermal emissions that occur in the Subpelagonian and Vardar/ Axios zones (IH) plot in the field characteristic for abiogenic hydrocarbons emitted from volcanic-geothermal systems after McCollom and
Seewald (2007) (Fig. 6a). At the same time, also the $\mathrm{CO}_{2}$-rich thermal manifestations from $\mathrm{HH}$ (Rhodope massif) and IH (Pelagonian and Subpelagonian zones) are found in a another field proposed by Sherwood Lollar et al. (2006) to be characteristic for an abiogenic origin, releasing gases mainly related to hydrothermal systems within the crystalline or metamorphic rocks of the Precambrian shield (Anders et al., 2006; Reischmann and Kostopoulos, 2007) (Fig. 6a). In both cases, a contribution from a biogenic source cannot be excluded due to the closeness to the thermogenic field. In the latter case however, the samples are found in low to medium enthalpy back-arc geothermal fields in an area characterised by extensional tectonics, which presumably also results in crust thinning (Fytikas and Kolios, 1979). $\mathrm{CO}_{2}$ dominated thermal gases from the VA show chemical and isotopic characteristics that are apparently consistent with an abiogenic origin for $\mathrm{CH}_{4}\left(\delta^{13} \mathrm{C}-\mathrm{CH}_{4}\right.$ values around $-20 \%$, and $\delta^{2} \mathrm{H}-\mathrm{CH}_{4}$ values around $-150 \%$ ) deriving from $\mathrm{CO}_{2}$ reduction. Contributions from a biogenic source cannot be excluded for those samples having $\mathrm{CH}_{4}$ / $\left(\mathrm{C}_{2} \mathrm{H}_{6}+\mathrm{C}_{3} \mathrm{H}_{8}\right)$ ratios $<1000$ (Bernard ratio ranges from 9 to 4810 ).

Some $\mathrm{N}_{2}$-rich gases from $\mathrm{IH}$ discharge in correspondence of the ophiolitic bodies that crop out in the Hellenic territory (Pe-Piper and Piper, 2002), where hyperalkaline waters ( $\mathrm{pH}$ from 9.72 to 11.98) were found at Othrys (central Greece - Etiope et al., 2013b; D'Alessandro et al., 2014) and at Argolida (D'Alessandro et al., 2017). $\mathrm{CH}_{4}$ collected in these hyperalkaline springs show $\delta^{13} \mathrm{C}_{-} \mathrm{CH}_{4}$ values ranging from -37.4 to $-26.6 \%$ and $\delta^{2} \mathrm{H}-\mathrm{CH}_{4}$ from -311 to $-250 \%$, excluding sample 88 that has more positive values $(-2.0$ and $-127 \%$ respectively) that were attributed by D'Alessandro et al. (2017) to microbial oxidation processes. Such values, on the diagram of Fig. $6 \mathrm{~b}$, fall all, except sample 88 , within the field of land-based serpentinization systems as defined by Etiope and Schoell (2014) and have been attributed by the previous authors to an abiogenic origin (Etiope et al., 2013b; D'Alessandro et al., 2014, 2017). 

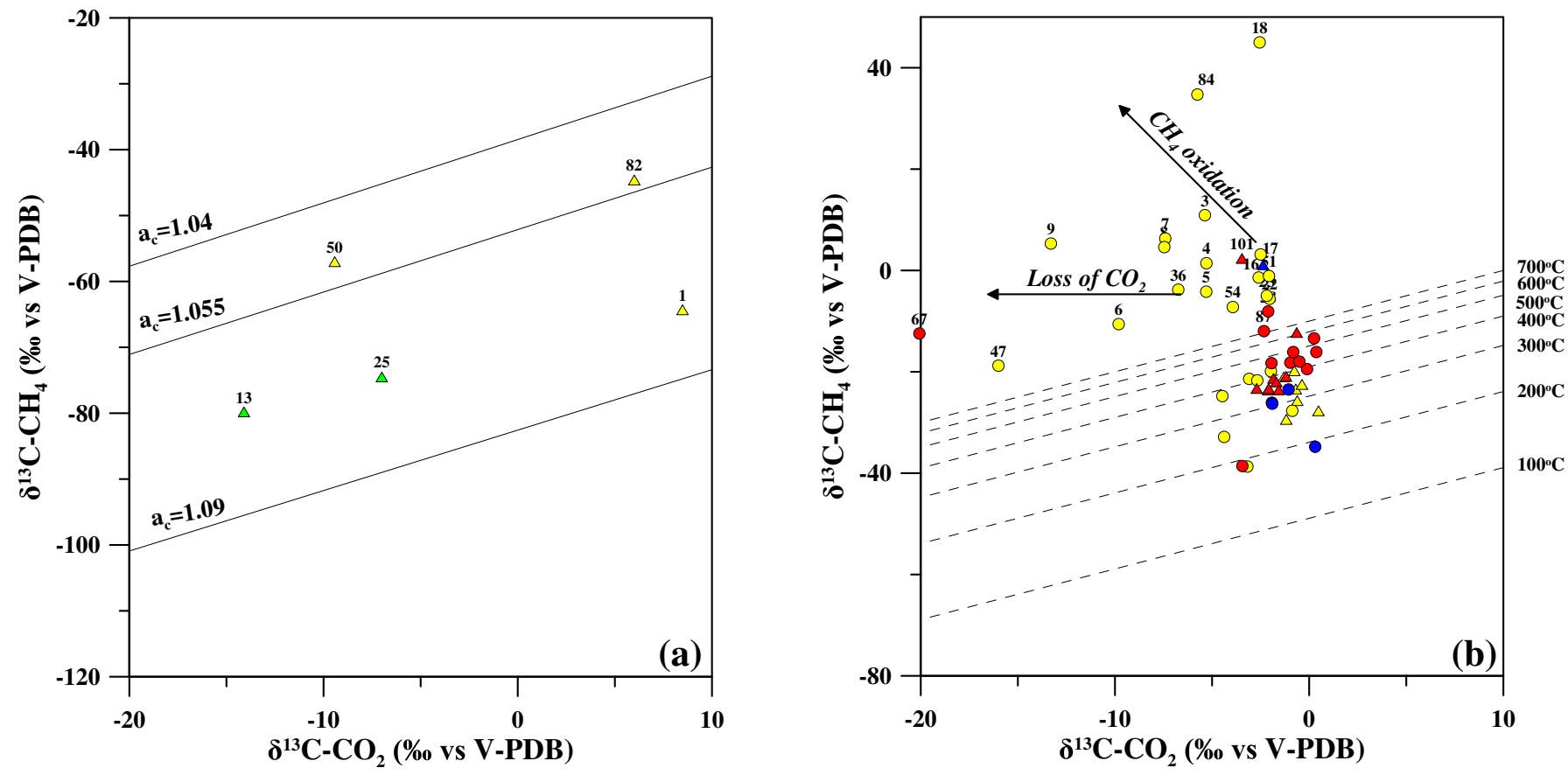

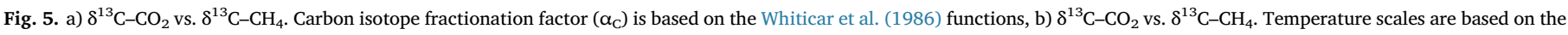
isotope fractionation factors from Bottinga (1969).
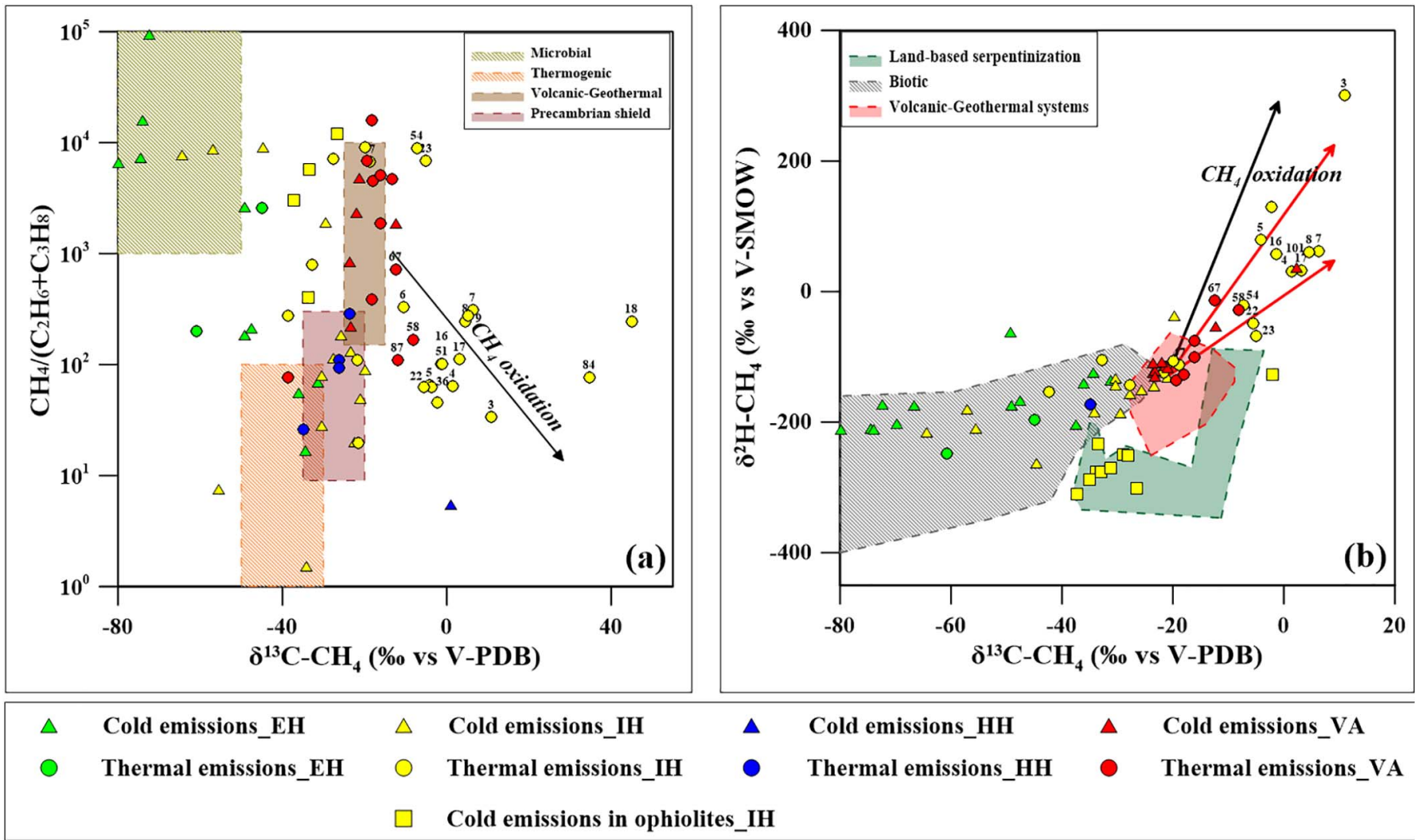

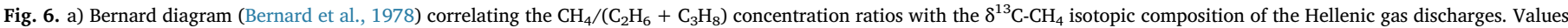

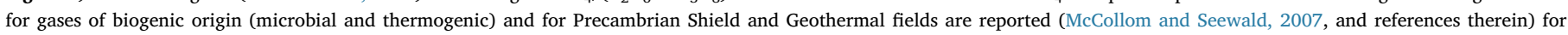

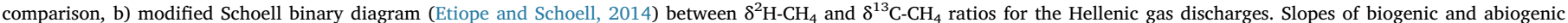

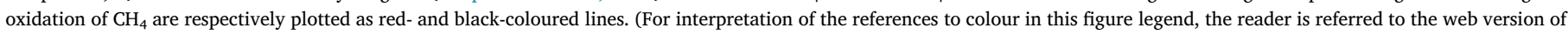
this article.) 


\subsection{Secondary post-genetic processes}

Many processes can significantly modify the isotopic signature of primary methane. Methanotrophic bacteria, for instance, may oxidize biogenic $\mathrm{CH}_{4}$, causing a shift toward less negative isotopic values of the residual gas (Coleman et al., 1981).

The majority of the thermal gases of Subpelagonian and Vardar/ Axios zones (IH), the cold manifestations of the Rhodope massif $(\mathrm{HH})$ and some of the volcanic-hydrothermal ones show low $\mathrm{CH}_{4} /$ $\left[\mathrm{C}_{2} \mathrm{H}_{6}+\mathrm{C}_{3} \mathrm{H}_{8}\right]$ ratios (Fig. 6a, Table 3 ) and strongly positive isotopic ratios of $\mathrm{CH}_{4}\left(\delta^{13} \mathrm{C}\right.$ up to $+45 \%$ and $\delta^{2} \mathrm{H}$ up to $+301 \%$ - Fig. $6 \mathrm{a}$, b, Table 2). Such chemical and isotopic features were likely caused by oxidation of $\mathrm{CH}_{4}$ (Fig. 5b). In these environments, microbes obtain energy from aerobic or anaerobic $\mathrm{CH}_{4}$ oxidation (Murrell and Jetten, 2009), preferentially consuming $\mathrm{CH}_{4}$ with respect to higher hydrocarbons and preferring light isotopes. Thermophilic and acidophilic methanotrophs oxidize $\mathrm{CH}_{4}$ also in the harsh environment of thermal waters up to temperatures of more than $80^{\circ} \mathrm{C}$ (Sharp et al., 2014). The temperatures of the sampling sites in which we found enriched $\delta^{13} \mathrm{C}$ and $\delta^{2} \mathrm{H}$ values for $\mathrm{CH}_{4}$ are mostly in the range from 40 to $63^{\circ} \mathrm{C}$ but reaching up to $73.7^{\circ} \mathrm{C}$ in the case of sample 54 . Although until now, no microbiological studies have been made on these waters to definitely support the occurrence of methanotrophs, the measured temperatures are well within the range that allows the presence of methane oxidizing microorganisms.

Inorganic oxidation of $\mathrm{CH}_{4}$ (Kiyosu and Imaizumi, 1996) in some samples cannot be ruled out. Nevertheless, the isotopic fractionations of organic and inorganic oxidation of $\mathrm{CH}_{4}$ follow different fractionation paths. The former follows $\Delta \mathrm{H} / \Delta \mathrm{C}$ slopes ranging from 5.9 to 13 (Cadieux et al., 2016 and references therein) and the latter a slope of 21 (Kiyosu and Imaizumi, 1996). Since in our samples it is not always possible to establish the primary isotopic composition before oxidation, we can only make some hypothesis about it. Looking at Fig. $6 \mathrm{~b}$ it is evident that most of the samples within the volcanic-geothermal field cluster around the following values: $\delta^{13} \mathrm{C} \approx-21 \%$ and $\delta^{2} \mathrm{H} \approx-130 \%$. Taking these values as the isotopic composition of $\mathrm{CH}_{4}$ before oxidation, we obtain $\Delta \mathrm{H} / \Delta \mathrm{C}$ values comprised between 3.8 and 13.6 mostly overlapping the typical range of biogenic oxidation processes. Furthermore, the strongly positive values shown by some samples imply low values of the residual fraction of $\mathrm{CH}_{4}(<0.25)$.

The relationship between $\delta^{13} \mathrm{C}$ values of $\mathrm{CO}_{2}$ and $\mathrm{CH}_{4}$ can be used to obtain useful information about the origin of these gas compounds (Whiticar et al., 1986). Assuming the attainment of an isotopic equilibrium between $\mathrm{CH}_{4}$ and $\mathrm{CO}_{2}$, equilibrium temperatures between the two gases can be computed according to Bottinga (1969) and Horita (2001). The estimation of the reservoir temperature through these geothermometers is outside the scope of this work. Nevertheless, most gases from VA and some thermal samples from $\mathrm{IH}$ and $\mathrm{HH}$ cover an estimated temperature interval between 270 and $500{ }^{\circ} \mathrm{C}$ indicating the possible achievement of isotopic equilibrium between $\mathrm{CH}_{4}$ and $\mathrm{CO}_{2}$. Among these, only for samples collected at Nisyros fumaroles Fiebig et al. $(2004,2007)$ demonstrated the attainment of equilibrium through the comparison with chemical geothermometers and temperatures measured in exploration wells. For all other samples, though indicating temperatures that are reasonable for geothermal systems, there is no proof for carbon isotope equilibration and the estimations may be likely only fortuitous. Instead all the samples plotting above the $700{ }^{\circ} \mathrm{C}$ isotherm are likely affected by secondary processes that isotopically fractionate $\mathrm{CO}_{2}$ and $\mathrm{CH}_{4}$. Such processes, as evidenced previously, are the $\mathrm{CO}_{2}$ loss and the microbially-driven $\mathrm{CH}_{4}$ oxidation. The former affects only the $\delta^{13} \mathrm{C}-\mathrm{CO}_{2}$ through either gas dissolution in water or precipitation as carbonate, whereas the latter tends toward more positive $\delta^{13} \mathrm{C}-\mathrm{CH}_{4}$ values and more negative $\delta^{13}{\mathrm{C}-\mathrm{CO}_{2}}_{2}$ values (Fig. $5 \mathrm{~b}$ ).

As shown in Fig. 7 , the $\mathrm{CH}_{4} / \mathrm{C}_{2} \mathrm{H}_{6}$ concentration ratios of the gas emissions vary by more than four orders of magnitude. On the contrary, the ratios between the main light hydrocarbons, i.e. $\mathrm{C}_{2} \mathrm{H}_{6}, \mathrm{C}_{3} \mathrm{H}_{8}$ and

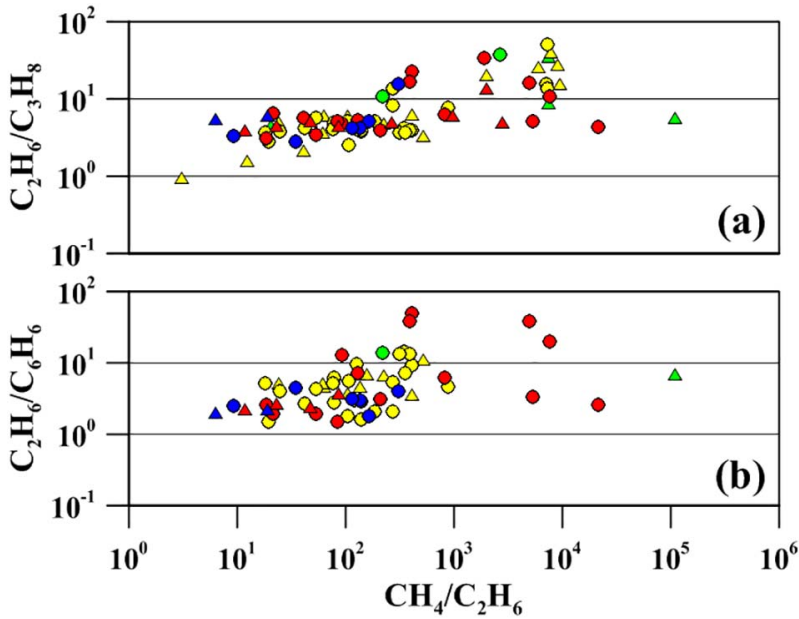

Fig. 7. a) $\mathrm{CH}_{4} / \mathrm{C}_{2} \mathrm{H}_{6}$ vs. $\mathrm{C}_{2} \mathrm{H}_{6} / \mathrm{C}_{3} \mathrm{H}_{8}$ and b) $\mathrm{CH}_{4} / \mathrm{C}_{2} \mathrm{H}_{6}$ vs. $\mathrm{C}_{2} \mathrm{H}_{6} / \mathrm{C}_{6} \mathrm{H}_{6}$ binary diagrams for the Hellenic gas discharges. Symbols as in Fig. 3.

$\mathrm{C}_{6} \mathrm{H}_{6}$, vary only within two orders of magnitude. In this respect, the $\mathrm{CO}_{2}(\mathrm{CO})-\mathrm{CH}_{4}$ interaction that likely controls the $\mathrm{CH}_{4}$ abundance in volcanic fluids does not seem to affect the higher hydrocarbons whose origin could be entirely related to thermal degradation of organic matter (Fiebig et al., 2009, 2015).

$\mathrm{CH}_{4}$ polymerization is accompanied by relatively small carbon isotope fractionations between $\mathrm{C}_{2+} n$-alkanes and residual $\mathrm{CH}_{4}$ (Sherwood Lollar et al., 2006; McCollom et al., 2010). This hypothesis should be supported by C-isotope data on $\mathrm{C}_{2}+$ alkane series' that unfortunately are not available for this study. Likewise, the residual $\mathrm{CH}_{4}$ retains its primary carbon isotopic composition if the degree of polymerization is low. This, alternatively, might provide an explanation why there occurs no significant correlation between the ${ }^{13} \mathrm{C}$ composition of $\mathrm{CH}_{4}$ and the magnitude of the Bernard ratio values for gases from VA (Fig. 6a), with $\delta^{13} \mathrm{C}-\mathrm{CH}_{4}$ being entirely controlled by $\delta^{13} \mathrm{C}-\mathrm{CO}_{2}$ and temperature.

\section{Conclusions}

Our results show that $\mathrm{CH}_{4}$ from the Hellenic territory describes a wide range of both concentrations (from $<0.5$ to $925,200 \mu \mathrm{mol} / \mathrm{mol}$ ) and isotopic values $\left(\delta^{13} \mathrm{C}-\mathrm{CH}_{4}\right.$ from -79.8 to $+45.0 \%$; $\delta^{2} \mathrm{H}_{-} \mathrm{CH}_{4}$ from -311 to $+301 \% 0$ ). Furthermore, the $\mathrm{CH}_{4} /\left(\mathrm{C}_{2} \mathrm{H}_{6}+\mathrm{C}_{3} \mathrm{H}_{8}\right)$ concentration ratio displays a broad range of values $(1.5-93,200)$. Such a large variability in hydrocarbon concentration ratios and methane isotopic compositions is indicative for methane originating from different sources and for the importance of secondary, post genetic processes such as microbial oxidation. A schematic concluding description of the results combined with the variations of the geology, the heat flow values, and $\mathrm{CO}_{2}$ and $\mathrm{CH}_{4}$ concentrations along the Hellenic territory is proposed in the summary flow chart diagram (Fig. 8).

Taking into account the different lithological facies of the study area and the dominant gas species, it is noticeable that samples found on the western part of Greece (EH) display higher $\mathrm{CH}_{4}$ and $\mathrm{N}_{2}$ concentrations with respect to those found in the eastern part $(\mathrm{IH}, \mathrm{HH})$ where $\mathrm{CO}_{2}$ is the prevailing gas. This can be explained by the sedimentary regime that characterises the $\mathrm{EH}$, in which solid organic substances dominate, favouring the occurrence of hydrocarbon deposits. Moreover, the continuous changes in the relief and the mainly intrusive and metamorphic formations of both $\mathrm{IH}$ and $\mathrm{HH}$ contain few or no amounts of organic matter.

Biogenic methane was mainly found in the $\mathrm{N}_{2}-\mathrm{CH}_{4}$ and $\mathrm{CH}_{4}$-dominated gases from EH. More specifically, gases collected in the GavrovoTripolis zone show a dominating microbial origin. Gas samples of the Ionian and Pindos zones are produced by both microbial activity and thermal maturation of sedimentary organic matter. On the contrary 


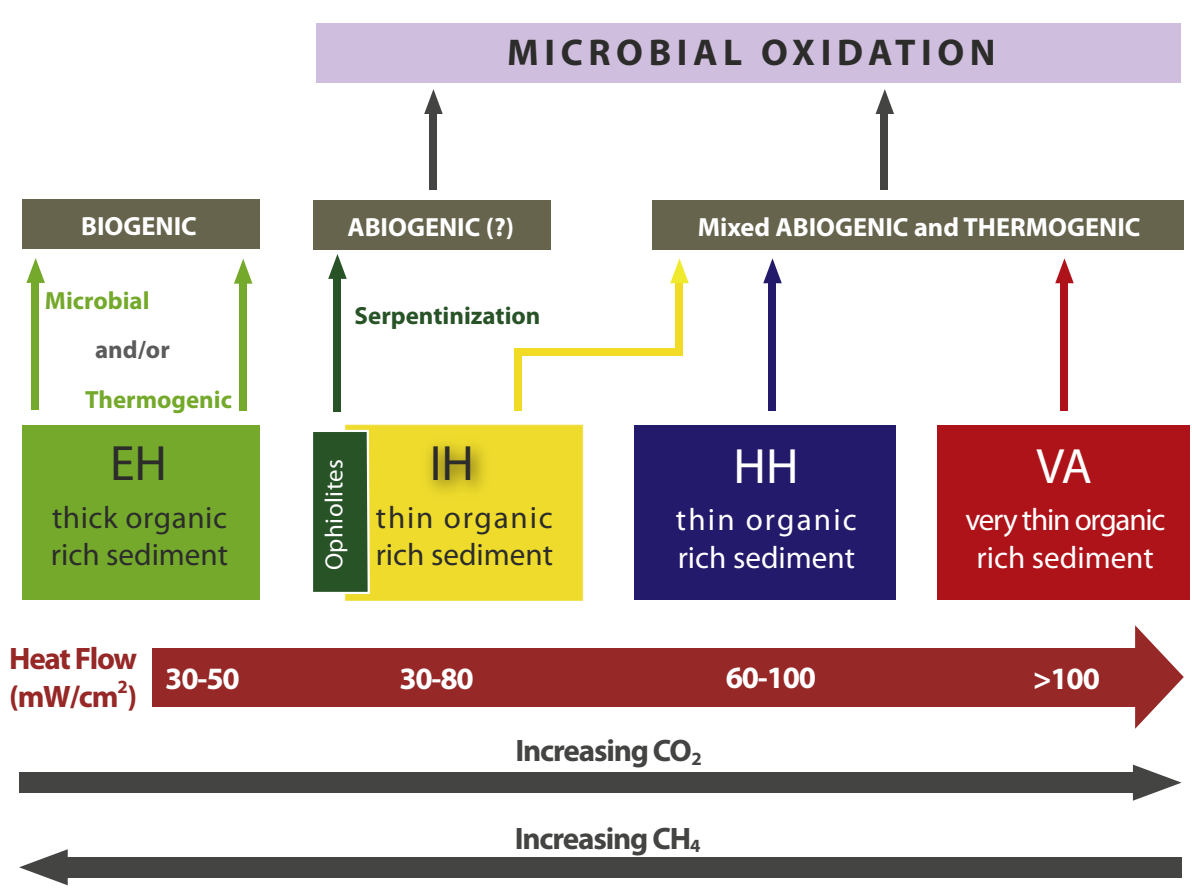

Fig. 8. A graphical description of the different possible origins of $\mathrm{CH}_{4}$ including postgenetic processes. Connectors are emerging from the central boxes to the possible origins and the processes that are affecting its origin. The main boxes provide information about the thickness of the sedimentary sequences in the different geologic regions. Both variations of heat flow values and $\mathrm{CO}_{2}$ and $\mathrm{CH}_{4}$ concentrations along the regions are plotted on the lower part of the flow chat. pure thermogenic samples are less represented in the sampled manifestations and are connected either to high geothermal gradients (samples 21 and 75 ) or to very thick sedimentary sequences in the Ionian (EH) and Aegean Sea (IH) (samples 104, 105 and 110 to 113).

Hydrocarbons contained in $\mathrm{CO}_{2}$-rich thermal manifestations from $\mathrm{HH}$ (Rhodope massif) and IH (Pelagonian and Subpelagonian zones) are - regarding their isotopic composition - similar to those emitted from the crystalline or metamorphic rocks of the Precambrian shield (Sherwood Lollar et al., 2006). They, therefore, may be considered of abiogenic origin. However, for most of these samples, the measured values could also be explained by a biogenic origin modified by methane oxidation processes.

$\mathrm{CO}_{2}$ dominated thermal gases from VA and $\mathrm{N}_{2}-\mathrm{CO}_{2}$-dominated thermal gases from $\mathrm{IH}$ show a relatively narrow range of $\delta^{13} \mathrm{C}-\mathrm{CH}_{4}$ values $\left(-25\right.$ to $-15 \%$ ) and a much larger range of $\mathrm{CH}_{4}$ / $\left(\mathrm{C}_{2} \mathrm{H}_{6}+\mathrm{C}_{3} \mathrm{H}_{8}\right)$ concentration ratios $(10$ to 10,000$)$ that show chemical and isotopic characteristics that are apparently consistent with an abiogenic origin for $\mathrm{CH}_{4}$ with a minor contribution of a thermogenic source. For the geothermal system of Nisyros, which belongs to the VA, $\mathrm{CH}_{4}$ is mostly originated from inorganic reactions in the hydrothermal reservoir while the light hydrocarbons have a prevailingly thermogenic origin (Fiebig et al., 2009).

Some of the $\mathrm{N}_{2}$-rich gas manifestations of the $\mathrm{IH}$ collected in Pindos zone, seem to have a possible abiogenic origin for $\mathrm{CH}_{4}$ as they are associated with the ophiolitic sequences of Othrys and Argolida. Finally, microbial oxidation processes have been evidenced for some of the $\mathrm{CO}_{2}$ dominated gas discharges from the main thermal springs located in the Subpelagonian and Vardar-Axios zones $(\mathrm{IH})$ and in the Rhodope massif $(\mathrm{HH})$. Such processes lead to sometimes very strong isotopic fractionation of $\mathrm{CH}_{4}$ reaching very positive $\delta^{13} \mathrm{C}(+45.0 \%)$ and $\delta^{2} \mathrm{H}$ $(+301 \%)$ values.

Finally, we want to underscore that, although further studies are necessary to answer a number of questions that remained open such as the accurate origin of hydrocarbons, the important contribution of this paper is to show how hydrocarbons from all over Greece distribute in the Schoell and Bernard plots. Considering this, our study may be a starting point for those who want to investigate the geochemistry of fluids from a specific area included in this study and to examine in more detail their origin by applying new scientific approaches, such as those based on clumped isotopes.
Supplementary data to this article can be found online at https:// doi.org/10.1016/j.chemgeo.2018.01.027.

\section{Acknowledgments}

We kindly acknowledge all the friends and colleagues that helped us either in the field or with precious information about the sampling sites. Among them we are indebted with Dr. Markos Xenakis and Dr. Konstantinos Athanasoulis of the IGME for their precious information about the location of many interesting sites all around the Hellenic territory. Also we would like to thank Francesco Capecchiacci (GC laboratory, UniFi), Mauro Martelli and Francesco Salerno (GC laboratory INGV-Pa), Ygor Oliveri and Aldo Sollami (MS laboratory INGV-Pa), Andrea Rizzo and Mariano Tantillo (Noble Gas laboratory INGV-Pa) for their kind and valuable support in the analyses and Silvia Eleonora Angileri and Stefano Dell'Aria for their useful help in drawing some of the figures. This work is part of the $\mathrm{PhD}$ thesis research of the first author $\mathrm{PhD}$ in Earth and Marine Sciences - University of Palermo, 30th cycle and PhD in Applied Environmental Geology - National and Kapodistrian University of Athens). We are grateful for the insightful comments of Andri Stefánsson and an anonymous referee and of the editor Hailiang Dong that helped us to significantly improve the manuscript. We are also indebted to David Hilton, who managed as editor the first round of review of the manuscript. We were deeply touched by the notice of his death. Many of us knew him personally and we would like to express all our admiration for his human and scientific qualities.

\section{References}

Anders, B., Reischmann, T., Kostopoulos, D., 2006. The oldest rocks of Greece: first evidence for a Precambrian terrane within Pelagonian Zone. Geol. Mag. 143, 41-58.

Barnes, I., O'Neil, J.R., 1969. The relationship between fluids in some fresh alpine type ultramafics and possible modern serpentinization, western United States. Geol. Soc. Am. Bull. 80, 1947-1960.

Bernard, B.B., Brooks, J.M., Sackett, W.M., 1978. A Geochemical Model for Characterization of Hydrocarbon Gas Sources in Marine Sediments. Offshore Technology Conference, Houston, USA, pp. 435-438.

Berndt, M.E., Allen, D.E., Seyfried, W.E., 1996. Reduction of $\mathrm{CO}_{2}$ during serpentinization of olivine at $300^{\circ} \mathrm{C}$ and 500 bar. Geology 24, 351-354.

Bortolotti, V., Principi, G., 2005. Tethyan ophiolites and Pangea break-up. Island Arc 14, $442-470$.

Bottinga, Y., 1969. Calculated fractionation factors for carbon and hydrogen isotope 
exchange in the system calcite-carbon dioxide-graphite-methane-hydrogen-water vapor. Geochim. Cosmochim. Acta 33, 49-64.

Bradley, A.S., Summons, R.E., 2010. Multiple origins of methane at the Lost City hydrothermal field. Earth Planet. Sci. Lett. 297, 34-41.

Bruni, J., Canepa, M., Cipolli, F., Marini, L., Ottonello, G., Vetuschi Zuccolini, M., et al., 2002. Irreversible water-rock mass transfer accompanying the generation of the neutral, $\mathrm{Mg}-\mathrm{HCO}_{3}$ and high-pH, Ca-OH spring waters of the Genova province, Italy. Appl. Geochem. 17, 455-474.

Burke, R.A., Barber, T., Sackett, W., 1988. Methane flux and stable H and C isotope composition of the sedimentary methane from the Florida Everglades. Glob. Biogeochem. Cycles 2, 329-340.

Burke, R.A., Martens, C.S., Sackettl, W., 1988. Seasonal variations of D/H and ${ }^{13} \mathrm{C} /{ }^{12} \mathrm{C}$ ratios of microbial methane in surface sediments. Nature 332, 829-831.

Burton, P., Xu, Y., Qin, C., Tselentis, G., Sokos, E., 2004. A catalogue of seismicity in Greece and the adjacent areas of the twentieth century. Tectonophysics 390, 117-127.

Cadieux, S.B., White, J.R., Sauer, P.E., Peng, Y., Goldman, A.E., Pratt, L.M., 2016. Large fractionations of $\mathrm{C}$ and $\mathrm{H}$ isotopes related to methane oxidation in Arctic lakes. Geochim. Cosmochim. Acta 187, 141-155.

Capaccioni, B., Martini, M., Mangani, F., Giannini, L., Nappi, G., Prati, F., 1993. Light hydrocarbons in gas-emissions from volcanic areas and geothermal fields. Geochem. J. 27, 7-17.

Capaccioni, B., Taran, Y., Tassi, F., Vaselli, O., Mangani, G., Macias, J.L., 2004. Source conditions and degradation processes of light hydrocarbons in volcanic gases: an example from El Chichon volcano (Chiapas State, Mexico). Chem. Geol. 206, 81-96.

Capasso, G., Inguaggiato, S., 1998. A simple method for the determination of dissolved gases in natural waters. An application to thermal waters from Vulcano Island. Appl. Geochem. 13, 631-642.

Cavazza, W., Roure, F., Spakman, W., Stampfli, G.M., Ziegler, P.A., 2014. The TRANSMED Atlas - The Mediterranean Region from Crust to Mantle Geological and Geophysical Framework of the Mediterranean and the Surrounding Areas. Springer, Verlag Berlin, Heidelberg, New York, NY.

Chanton, J.P., Chasar, L.C., Glaser, P., Siegel, D., 2005. Carbon and hydrogen isotopic effects in microbial methane from terrestrial environments. In: Flanagan, L.B., Ehleringer, J.R., Pataki, D.E. (Eds.), Stable Isotopes and Biosphere-Atmosphere Interactions. Physiol. Ecol. Ser., vol. 6. Elsevier, New York, pp. 85-105.

Chiodini, G., 1994. Temperature, pressure and redox conditions governing the composition of the cold $\mathrm{CO}_{2}$ gases discharged in the volcanic area of North Latium (Central Italy). Appl. Geochem. 9, 287-295.

Ciais, P., Sabine, C., Bala, G., Bopp, L., Brovkin, V., Canadell, J., Chabra, A., De Fries, R., Galloway, J., Heimann, M., Jones, C., Le Quéré, C., Myneni, R.B., Piao, S., Thornton, P., 2013. Carbon and other biogeochemical cycles. In: Stocker, T.F. (Ed.), Climate Change 2013: The Physical Science Basis. Contribution of Working Group I to the Fifth Assessment Report of IPCC. Cambridge University Press, Cambridge.

Coleman, D.D., Risatti, J.B., Schoell, M., 1981. Fractionation of carbon and hydrogen isotopes by methane-oxidizing bacteria. Geochim. Cosmochim. Acta 45, 1033-1037.

D'Alessandro, W., Brusca, L., Kyriakopoulos, K., Bellomo, S., Calabrese, S., 2014. A geochemical traverse along the "Sperchios Basin — Evoikos Gulf" Graben (Central Greece): origin and evolution of the emitted fluids. Mar. Pet. Geol. 55, 295-308. http://dx.doi.org/10.1016/j.marpetgeo.2013.12.011.

D'Alessandro, W., Daskalopoulou, K., Calabrese, S., Bellomo, S., 2017. Water chemistry and abiogenic methane content of a hyperalkaline spring related to serpentinization in the Argolida ophiolite (Ermioni, Greece). Mar. Pet. Geol. http://dx.doi.org/10. 1016/j.marpetgeo.2017.01.028.

D'Alessandro, W., Kyriakopoulos, K., 2013. Preliminary gas hazard evaluation in Greece. Nat. Hazards 69, 1987-2004.

Darling, W.G., 1998. Hydrothermal hydrocarbons gases: 1. Genesis and geothermometry. Appl. Geochem. 13, 815-824.

Dercourt, J., 1972. The Canadian cordillera, the Hellenides and the sea floor spreading theory. Can. J. Earth Sci. 9, 709-743.

Dimitrakopoulos, R., Muehlenbachs, K., 1987. Biodegradation of petroleum as a source of ${ }^{13} \mathrm{C}$ enriched carbon dioxide in the formation of carbonate cements. Chem. Geol. 65, 283-291.

Etiope, G., 2009. A Global Dataset of Onshore Gas and Oil Seeps: A New Tool for Hydrocarbon Exploration. Oil and Gas Business. http://ogbus.ru/eng/authors Etiope/Etiope_1.pdf (accessed 07/07/2017).

Etiope, G., 2015. Natural Gas Seepage. The Earth's Hydrocarbon Degassing. Springer International Publishing Switzerland, pp. 119. e-book. Ch 7.1.5. https://doi.org/10. 1007/978-3-319-14601-0.

Etiope, G., Sherwood Lollar, B., 2013. Abiotic methane on Earth. Rev. Geophys. 51, 276-299. http://dx.doi.org/10.1002/rog.20011.

Etiope, G., Schoell, M., 2014. Abiotic gas: atypical but not rare. Elements 10, 291-296.

Etiope, G., Ionescu, A., 2015. Low-temperature catalytic $\mathrm{CO}_{2}$ hydrogenation with geological quantities of ruthenium: a possible abiotic $\mathrm{CH}_{4}$ source in chromite-rich serpentinized rocks. Geofluids 15, 438-452.

Etiope, G., Papatheodorou, G., Christodoulou, D., Ferentinos, G., Sokos, E., Favali, P., 2006. Methane and hydrogen sulfide seepage in the NW Peloponnesus petroliferous basin (Greece): origin and geohazard. AAPG Bull. 90, 701-713.

Etiope, G., Caracausi, A., Favara, R., Italiano, F., Baciu, C., 2009. Methane emission from the mud volcanoes of Sicily (Italy). J. Geophys. Res. Lett. 29 (8), 1215.

Etiope, G., Christodoulou, D., Kordella, S., Marinaro, G., Papatheodorou, G., 2013a. Offshore and onshore seepage of thermogenic gas at Katakolo Bay (Western Greece). Chem. Geol. 339, 115-126.

Etiope, G., Tsikouras, B., Kordella, S., Ifandi, E., Christodoulou, D., Papatheodorou, G., 2013b. Methane flux and origin in the Othrys ophiolite hyperalkaline springs, Greece. Chem. Geol. 347, 161-174.

Ferrière, J., Baumgartner, P.O., Chanier, F., 2016. The Maliac Ocean: the origin of Tethyan Hellenic ophiolites. Int. J. Earth Sci. 105, 1941-1963. http://dx.doi.org/10. 1007/s00531-016-1303-6.

Fiebig, J., Chiodini, G., Caliro, S., Rizzo, A., Spandenberg, J., Hunziker, J.C., 2004.
Chemical and isotopic equilibrium between $\mathrm{CO}_{2}$ and $\mathrm{CH}_{4}$ in fumarolic gas discharges: generation of $\mathrm{CH}_{4}$ in arc magmatic-hydrothermal systems. Geochim. Cosmochim. Acta 68, 2321-2334.

Fiebig, J., Hofmann, S., Tassi, F., D'Alessandro, W., Vaselli, O., Woodland, A.B., 2015. Isotopic patterns of hydrothermal hydrocarbons emitted from Mediterranean volcanoes. Chem. Geol. 396, 152-163. http://dx.doi.org/10.1016/j.chemgeo.2014.12. 030 .

Fiebig, J., Tassi, F., D'Alessandro, W., Vaselli, O., Woodland, A., 2013. Carbon-bearing gas geothermometers for volcanic-hydrothermal systems. Chem. Geol. 351, 66-75.

Fiebig, J., Woodland, A., D'Alessandro, W., Puttmann, W., 2009. Excess methane in continental hydrothermal emissions is abiogenic. Geology 37, 495-498.

Fiebig, J., Woodland, A.B., Spangenberg, J., Oschmann, W., 2007. Natural evidence for rapid abiogenic hydrothermal generation of $\mathrm{CH}_{4}$. Geochim. Cosmochim. Acta 71, 3028-3039.

Fischer, F., Tropsch, H., 1923. The preparation of synthetic oil mixtures (synthol) from carbon monoxide and hydrogen. Brennst. Chem. 4, 276-285.

Fischer, F., Tropsch, H., 1926. Über die direkte Synthese von Eerdöl-kohlenwasserstoffen bei gewöhnlichtem Druck. Ber. Dtsch. Chem. Ges. 59, 830-831.

Formolo, M., 2010. The microbial production of methane and other volatile hydrocarbons. In: Kenneth, N. (Ed.), Timmis Handbook of Hydrocarbon and Lipid Microbiology. Springer, New York, pp. 113-126.

Foustoukos, D.I., Seyfried, W.E., 2004. Hydrocarbons in hydrothermal vent fluids: the role of chromium-bearing catalysts. Science 304, 1002-1004.

Fytikas, M., Innocenti, F., Kolios, N., Manetti, P., Mazzuoli, R., 1986. The Plio-Quaternary volcanism of the Saronikos area (western part of the active Aegean volcanic arc). Ann. Géol. Pays Hellén. 33, 23-45.

Fytikas, M., Kolios, N., 1979. Preliminary heat flow map of Greece. In: Cermak, V., Rybach, L. (Eds.), Terrestrial Heat Flow in Europe. Springer-Verlag, Berlin Heidelberg New York, pp. 197-205.

Giggenbach, W.F., 1980. Geothermal gas equilibria. Geochim. Cosmochim. Acta 44, 2021-2032.

Giggenbach, W.F., 1987. Redox processes governing the chemistry of fumarolic gas discharges from White Island, New Zealand. Appl. Geochem. 2, 143-161.

Giggenbach, W.F., 1996. Chemical composition of volcanic gases. In: Scarpa, R., Tilling, R.I. (Eds.), Monitoring and Mitigation of Volcano Hazards. Springer, pp. 221-256.

Giggenbach, W.F., Gougel, R.L., 1989. Method for the collection and analysis of geothermal and volcanic water and gas samples. NZ-DSIR Report CD 2387, 53.

Grassa, F., Capasso, G., Oliveri, Y., Sollami, A., Carreira, P., Rosário Carvalho, M., Marques, J.M., Nunes, J.C., 2010. Nitrogen isotopes determination in natural gas: analytical method and first results on magmatic, hydrothermal and soil gas samples. Isot. Environ. Health Stud. 46, 141-155.

Grigoriadis, V., Tziavos, I., Tsokas, G., Stampolidis, A., 2016. Gravity data inversion for Moho depth modeling in the Hellenic area. Pure Appl. Geophys. 173, 1223-1241. 2015 Springer Basel DOI. https://doi.org/10.1007/s00024-015-1174-y.

Guliyev, I.S., Feizullayev, A.A., 1997. All about mud volcanoes. Baku Pub. House, NAFTAPress (120 pp.).

Gunter, B.D., 1978. C1-C4 hydrocarbons in geothermal gases. Geochim. Cosmochim. Acta 42, 137-139.

Hinrichs, K.U., Hayes, J.M., Bach, W., Spivack, A.J., Hmelo, L.R., Holm, N.G., Johnson, C.G., Sylva, S.P., 2006. Biological formation of ethane and propane in the deep marine subsurface. Proc. Natl. Acad. Sci. 103 (40), 14684-14689. http://dx.doi.org/ 10.1073/pnas.0606535103.

Holloway, J.R., 1984. Graphite- $\mathrm{CH}_{4}-\mathrm{H}_{2} \mathrm{O}-\mathrm{CO}_{2}$ equilibria at low-grade metamorphic conditions. Geology 12, 455-458.

Horita, J., 2001. Carbon isotope exchange in the system $\mathrm{CO}_{2}-\mathrm{CH}_{4}$ at elevated temperatures. Geochim. Cosmochim. Acta 65, 1907-1919.

Horita, J., Berndt, M.E., 1999. Abiogenic formation and isotopic fractionation under hydrothermal conditions. Science 285, 1055-1057.

Hornibrook, E.R.C., Longstaffe, F.J., Fyfe, W.S., 1997. Spatial distribution of microbia methane production pathways in temperate zone wetland soils: stable carbon and hydrogen isotope evidence. Geochim. Cosmochim. Acta 61 (4), 745-753.

Hunt, J.M., 1996. Petroleum Geochemistry and Geology, 2nd ed. W.H. Freeman Co., New York (743 pp.).

Jenden, P.D., Hilton, D.R., Kaplan, I.R., Craig, H., 1993. Abiogenic hydrocarbons and mantle helium in oil and gas fields. In: Howell, D.G. (Ed.), The Future of Energy Gases: US Geol. Surv. Profes. Paper. vol. 1570. pp. 31-56.

Kamberis, E., Rigakis, N., Tsaila-Monopolis, S., Ioakim, C., Sotiropoulos, S., 2000. Shallow biogenic gas-accumulations in late Cenozoic sands of Katakolon Peninsula, western Greece. Geol. Soc. Greece, Spec. Publ. 9, 121-138.

Kanellopoulos, C., 2012. Distribution, lithotypes and mineralogical study of newly formed thermogenic travertines in Northern Euboea and Eastern Central Greece. Cent. Eur. J. Geosci. 4 (4), 545-560. http://dx.doi.org/10.2478/s13533-012-0105-z.

Kanellopoulos, C., Mitropoulos, P., Valsami-Jones, E., Voudouris, P., 2017. A new ter restrial active mineralizing hydrothermal system associated with ore-bearing travertines in Greece (northern Euboea Island and Sperchios area). J. Geochem. Explor. $179,9-24$.

Kietäväinen, R., Purmako, L., 2015. The origin, source, and cycling of methane in deep crystalline rock biosphere. Front. Microbiol. 6, 725

Kinnaman, F.S., Valentine, D.L., Tyler, S.C., 2007. Carbon and hydrogen isotope fractionation associated with the aerobic microbial oxidation of methane, ethane, propane and butane. Geochim. Cosmochim. Acta 71, 271-283.

Kiyosu, Y., Imaizumi, S., 1996. Carbon and hydrogen isotope fractionation during oxidation of methane by metal oxides at temperatures from $400^{\circ}$ to $530^{\circ} \mathrm{C}$. Chem. Geol. 133, 279-287.

Klusman, R.W., Jakel, M.E., 1998. Natural microseepage of methane to the atmosphere from the Denver-Julesburg basin, Colorado, USA. J. Geophys. Res. 103D, $28,042-28,045$.

Konn, C., Charlou, J.L., Holm, N.G., Mousis, O., 2015. The production of methane, hydrogen, and organic compounds in ultramafic- hosted hydrothermal vents of the MidAtlantic Ridge. Astrobiology 15 (5), 381-399. 
Kvenvolden, K.A., 1993. Gas hydrates—geological perspective and global change. Rev. Geophys. 31, 173-187.

Machel, H.G., Krouse, H.R., Sassen, R., 1995. Products and distinguishing criteria of bacterial and thermochemical sulphate reduction. Appl. Geochem. 10, 373-389.

Mango, F.D., 2000. The origin of light hydrocarbons. Geochim. Cosmochim. Acta 64 1265-1277.

Marini, L., Fiebig, J., 2005. Fluid geochemistry of the magmatic-hydrothermal system of Nisyros (Greece). Memoires Geol. (Lausanne) 44, 121-163.

McCollom, T.M., 2013. Laboratory simulations of abiotic hydrocarbon formation in Earth's deep subsurface. Rev. Mineral. Geochem. 75, 467-494.

McCollom, T.M., Seewald, J.S., 2007. Abiotic synthesis of organic compounds in deep-sea hydrothermal environments. Chem. Rev. 107, 382-401.

McCollom, T.M., Sherwood Lollar, B., Lacrampe-Couloume, G., Seewald, J.S., 2010. The influence of carbon source on abiotic organic synthesis and carbon isotope fractionation under hydrothermal conditions. Geochim. Cosmochim. Acta 74, 2717-2740.

Milkov, A.V., 2000. Worldwide distribution of submarine mud volcanoes and associated gas hydrates. Mar. Geol. 167, 29-42.

Miller, H.M., Mayhew, L.E., Ellison, E.T., Kelemen, P., Kubo, M., Templeton, A.S., 2017. Low temperature hydrogen production during experimental hydration of partiallyserpentinized dunite. Geochim. Cosmochim. Acta 209, 161-183.

Minissale, A., 2004. Origin, transport and discharge of $\mathrm{CO}_{2}$ in central Italy. Earth Sci. Rev. 66, 89-141.

Mörner, N.A., Etiope, G., 2002. Carbon degassing from the lithosphere. Glob. Planet Chang. 33, 185-203.

Mountrakis, D.M., 1985. Geologia tis Elladas. University Studio Press, Thessaloniki, pp. 207 (in Greek).

Mountrakis, D.M., 1986. The Pelagonian zone in Greece: a Polyphase-deformed fragment of the Cimmerian continent and its role in the geotectonic evolution of the Eastem Mediterranean. J. Geophys. 94, 335-347.

Mountrakis, D.M., 2010. Geologia kai Geotektoniki Exelixi tis Elladas. University Studio Press, Thessaloniki, pp. 374 (in Greek).

Murrell, C.J., Jetten, M.S.M., 2009. The microbial methane cycle. Environ. Microbiol. Rep. 1, 279-284.

Palacas, J.G., Monopolis, D., Nicolaou, C.A., Anders, D.E., 1986. Geochemical correlation of surface and subsurface oils, western Greece. Org. Geochem. 10, 417-423.

Pallasser, R.J., 2000. Recognising biodegradation in gas/oil accumulations through the $\delta^{13}$ compositions of gas components. Org. Geochem. 31, 1363-1373.

Paonita, A., Caracausi, A., Iacono-Marziano, G., Martelli, M., Rizzo, A., 2012. Geochemical evidence for mixing between fluids exsolved at different depths in the magmatic system of Mt Etna (Italy). Geochim. Cosmochim. Acta 84, 380-394.

Papanikolaou, D., 2009. Timing of tectonic emplacement of the ophiolites and terrane paleogeography in the Hellenides. Lithos 108, 262-280.

Pe-Piper, G., Piper, D.J.W., 2002. The igneous rocks of Greece, The anatomy of an orogen. In: Beiträge Zur Regionalen Geologie der Erde. vol. 30 Gebrüder Bornträger, Berlin Stuttgart.

Prinzhofer, A.A., Battani, A., 2003. Gas isotopes tracing: an important tool for hydrocarbon exploration. Oil Gas Sci. Technol. 58, 229-311.

Proskurowski, G., Lilley, M., Seewald, J.S., Fruh-Green, G.I., Olson, E.J., Sylva, S.P., Kelley, D.S., 2008. Abiogenic hydrocarbon production at Lost City hydrothermal field. Science 319, 604-607.

Proskurowski, G., Lilley, M.D., Kelley, D.S., Olson, E.J., 2006. Low temperature volatile production at the Lost City hydrothermal field, evidence from a hydrogen stable isotope geothermometer. Chem. Geol. 229, 331-343.

Quingley, T.M., MacKenzie, A.S., 1988. The temperatures of oil and gas formation in the sub-surface. Nature 333, 549-552.

Reischmann, T., Kostopoulos, D., 2007. Terrane accretion in the internal Hellenides. Geophys. Res. Abstr. 9, 05337.

Rigakis, N., Roussos, N., Kamberis, E., Proedrou, P., 2001. Hydrocarbon gas accumulations in Greece and their origin. Bull. Geol. Soc. Greece 34 (3), 1265-1273.

Rizzo, A.L., Barberi, F., Carapezza, M.L., Di Piazza, A., Francalanci, L., Sortino, F., D'Alessandro, W., 2015. New mafic magma refilling a quiescent volcano: evidence from He-Ne-Ar isotopes during the 2011-2012 unrest at Santorini, Greece. Geochem. Geophys. Geosyst. 16. http://dx.doi.org/10.1002/2014GC005653.

Roberts, G.P., Koukouvelas, L., 1996. Structural and seismological segmentation of the Gulf of Corinth fault system: implications for models of fault growth. Ann. Geophys. 39 (3), 619-646.

Robertson, A.H.F., 2004. Development of concepts concerning the genesis and emplacement of Tethyan ophiolites in the Eastern Mediterranean and Oman regions. EarthSci. Rev. 66, 331-387.

Robertson, A.H.F., 2012. Late Palaeozoic-Cenozoic tectonic development of Greece and Albania in the context of alternative reconstructions of Tethys in the Eastern Mediterranean region. Int. Geol. Rev. 54, 373-454.

Robertson, A.H.F., Dixon, J.F., 1984. Introduction: aspects ofthe geological evolution ofthe Eastem Mediterranean. In: Dixon, J.E., Robertson, A.H.F. (Eds.), The Geological Evolution of the Eastern Mediterranean. Geol. Soc. London Spec. Publ. Vol. 17. pp. $1-74$.

Sano, Y., Marty, B., 1995. Origin of carbon in fumarolic gas from island arcs. Chem. Geol. 119, 265-274.

Schoell, M., 1980. The hydrogen and carbon isotopic composition of methane from natural gases of various origins. Geochim. Cosmochim. Acta 44, 649-661.

Schoell, M., 1988. Multiple origins of methane in the Earth. Chem. Geol. 71, 1-10.

Seewald, J.S., 2001. Aqueous geochemistry of low molecular weight hydrocarbons at elevated temperatures and pressures. Constraints from mineral buffered laboratory experiments. Geochim. Cosmochim. Acta 65, 1641-1664.
Sharp, C.E., Smirnova, A.V., Graham, J.M., Stott, M.B., Khadka, R., Moore, T.R., Grasby, S.E., Strack, M., Dunfield, P.F., 2014. Distribution and diversity of Verrucomicrobia methanotrophs in geothermal and acidic environments. Environ. Microbiol. 16, 1867-1878.

Sherwood Lollar, B., Lacrampe-Couloume, G., Slater, G.F., Ward, J.A., Moser, D.P., Gihring, T.M., Lin, L.H., Onstott, T.C., 2006. Unravelling abiogenic and biogenic sources of methane in the Earth's deep subsurface. Chem. Geol. 226, 328-339.

Smith, A.G., Moores, E.M., 1974. Hellenides. Geol. Soc. Lond. Spec. Publ. 4, 159-185.

Snyder, G., Poreda, R.J., Fehn, U., Hunt, A., 2003. Sources of nitrogen and methane in Central American geothermal settings: noble gas and ${ }^{129} \mathrm{I}$ evidence for crustal and magmatic volatile components. Geochem. Geophys. Geosyst. 4. http://dx.doi.org/10. 1029/2002GC000363.

Snyder, G., Poreda, R.J., Hunt, A., Fehn, U., 2001. Regional variations in volatile composition: isotopic evidence for carbonate recycling in the Central American volcanic arc. Geochem. Geophys. Geosyst. 2. http://dx.doi.org/10.1029/2001GC 000163.

Stampfli, G., Vavassis, I., De Bono, A., Rosselet, F., Matti, B., Bellini, M., 2003. Remnants of the Paleotethys oceanic suture-zone in the western Tethyan area. Boll. Soc. Geol. Ital., Spec. 2, 1-23.

Stefánsson, A., Lemke, K.H., Bènèzeth, P., Schott, J., 2017. Magnesium bicarbonate and carbonate interactions in aqueous solutions: an infrared spectroscopic and quantum chemical study. Geochim. Cosmochim. Acta 198, 271-284.

Stefánsson, A., Sveinbjörnsdòttir, A.E., Heinemeier, J., Arnòrsson, S., Kjartansdòttir, R., Kristmannsdòttir, $\mathrm{H} ., 2$ 2016. Mantle $\mathrm{CO}_{2}$ degassing through the Icelandic crust: evidence from carbon isotopes in groundwater. Geochim. Cosmochim. Acta 191, 300-319.

Stolper, D.A., Lawson, M., Davis, C.L., Ferreira, A.A., Santos Neto, E.V., Ellis, G.S., Lewan, M.D., Martini, A.M., Tang, Y., Schoell, M., Sessions, A.L., Eiler, J.M., 2014. Gas formation. Formation temperatures of thermogenic and biogenic methane. Science 344 , 1500-1503.

Takai, K., Nakamura, K., Toki, T., Tsunogai, U., Miyazaki, M., Miyazaki, J., Hirayama, H., Nakagawa, S., Nunoura, T., Horikoshi, K., 2008. Cell proliferation at $122^{\circ} \mathrm{C}$ and isotopically heavy $\mathrm{CH}_{4}$ production by a hyperthermophilic methanogen under high pressure cultivation. Proc. Natl. Acad. Sci. 105, 10949-10954.

Taran, Y., Giggenbach, W., 2003. Geochemistry of light hydrocarbons in subduction-related volcanic and hydrothermal fluids. Society of Economic Geologists Special Publication 10 (6), 61-74.

Tassi, F., 2004. Fluidi in ambiente vulcanico: Evoluzione temporale dei parametri composizionali e distribuzione degli idrocarburi leggeri in fase gassosa (in Italian), Ph.D. thesis. Univ. of Florence, Florence, Italy, pp. 292.

Tassi, F., Fiebig, J., Vaselli, O., Nocentini, M., 2012. Origins of methane discharging from volcanic-hydrothermal, geothermal and cold emissions in Italy. Chem. Geol. 310-311, 36-48.

Tassi, F., Martinez, C., Vaselli, O., Capaccioni, B., Viramonte, J., 2005a. The light hydrocarbons as new geoindicators of equilibrium temperatures and redox conditions of geothermal fields: evidence from El Tatio (northern Chile). Appl. Geochem. 20, 2049-2062.

Tassi, F., Vaselli, O., Capaccioni, B., Giolito, C., Duarte, E., Fernandez, E., Minissale, A. Magro, G., 2005b. The hydrothermal-volcanic system of Rincon de la Vieja volcano (Costa Rica): a combined (inorganic and organic) geochemical approach to under standing the origin of the fluid discharges and its possible application to volcanic surveillance. J. Volcanol. Geotherm. Res. 148, 315-333.

Tilley, B., Muehlenbachs, K., 2013. Isotope reversals and universal stages and trends of gas maturation in sealed, self-contained petroleum systems. Chem. Geol. 339 (2013), 194-204.

van Hinsbergen, D.J.J., Hafkenscheid, E., Spakman, W., Meulenkamp, J.E., Wortel, M.J.R., 2005. Nappe stacking resulting from subduction of oceanic and continental lithosphere below Greece. Geology 33, 325-328.

Vaselli, O., Tassi, F., Montegrossi, G., Capaccioni, B., Giannini, L., 2006. Sampling and analysis of volcanic gases. Acta Vulcanol. 18, 65-76.

Wang, D.T., Gruen, D.S., Lollar, B.S., Hinrichs, K.U., Stewart, L.C., Holden, J.F., Hristov, A.N., Pohlman, J.W., Morrill, P.L., Könneke, M., Delwiche, K.B., Reeves, E.P., Sutcliffe, C.N., Ritter, D.J., Seewald, J.S., McIntosh, J.C., Hemond, H.F., Kubo, M.D., Cardace, D., Hoehler, T.M., Ono, S., 2015. Methane cycling. Nonequilibrium clumped isotope signals in microbial methane. Science 348 (6233), 428-431.

Welhan, J.A., 1988. Origins of methane in hydrothermal systems. Chem. Geol. 71, 183-198.

Welhan, J.A., Craig, H., 1983. Methane, hydrogen and helium in hydrothermal fluids of $21{ }^{\circ} \mathrm{N}$ on the East Pacific Rise. In: Rona, P.A., Boström, K., Laubier, L., Smith Jr.K.L. (Eds.), Hydrothermal Processes at Seafloor Spreading Centers. Plenum, New York, N.Y., pp. 391-409.

Whiticar, M.J., 1999a. Stable isotope geochemistry of coals, humic kerogen and related natural gases. Int. J. Coal Geol. 32, 191-215.

Whiticar, M.J., 1999b. Carbon and hydrogen isotope systematics of bacterial formation and oxidation of methane. Chem. Geol. 161, 291-314.

Whiticar, M.J., Faber, E., Schoell, M., 1986. Biogenic methane formation in marine and freshwater environments: $\mathrm{CO}_{2}$ reduction vs. acetate fermentation - isotopic evidence. Geochim. Cosmochim. Acta 50, 693-709.

Whiticar, M.J., Suess, E., 1990. Hydrothermal hydrocarbon gases in the sediments of the King-George Basin, Bransfield Strait, Antarctica. Appl. Geochem. 5, 135-147.

Winkel, L.H.E., Casentini, B., Bardelli, F., Voegelin, A., Nikolaidis, N.P., Charlet, L., 2013 Speciation of arsenic in Greek travertine: co-precipitation of arsenate with calcite. Geochim. Cosmochim. Acta 106, 99-110.

Worden, R.H., Smalley, P.C., 1996. $\mathrm{H}_{2}$ S-producing reactions in deep carbonate gas reservoirs: Khuff Formation, Abu Dhabi. Chem. Geol. 133, 157-171. 